\title{
1 Analysis of two-component non-equilibrium model of linear reactive
} 2 chromatography

3 Shamsul Qamar, Sameena Bibi, Noreen Akram and

4 Andreas Seidel-Morgenstern

6 the date of receipt and acceptance should be inserted later

7 Abstract This article presents semi-analytical solutions and analytical temporal moments of a two-components linear reactive lumped kinetic model incorporating irreversible $(A \rightarrow B)$ and reversible $(A \leftrightarrows B)$ reactions in a fixed-bed liquid chromatographic column. Both solid and liquid phase reactions and two sets of boundary conditions are considered. The current model equations contain a coupled system of two partial differential equations (PDEs) and two ordinary differential equations (ODEs). The solution methodology successively employs the Laplace transform and linear transformation steps to uncouple the governing set of coupled differential equations. The resulting system of uncoupled ODEs is solved by applying an elementary solution technique. The numerical Laplace inversion is employed to transform back the solutions in the actual time domain. To further analyze the effects of different kinetic parameters, statistical temporal moments are derived from the Laplace transformed solutions. The current solutions extend and generalize our recent solutions for single-solute transport models of non-reactive liquid chromatography. For verification, the analytical results are compared with the numerical solutions of a high resolution finite volume scheme. Several case studies of practical interest are considered. Good agreements in the results validate the correctness of semi-analytical solutions and the accuracy of proposed numerical algorithm.

Keywords Liquid chromatography, non-equilibrium transport, irreversible and reversible reactions, mass transfer, analytical solutions, moment analysis.

\section{1 introduction}

Column liquid chromatography is one of the most effective means of separation which is mainly used for the separation of components employed as fine chemicals, pharmaceuticals, food additives and biological products. The demand for efficient preparative and large-scale liquid chromatographic separation processes is ever increasing. The concept is successfully applied to perform numerous difficult separation processes, for example the separation of enantiomers and the isolation of specific proteins from fermentation broths. In the column liquid chromatography, a mobile phase percolates through a bed of fixed porous particles, carrying the mixture components which

S. Qamar, N. Akram

Department of Mathematics, COMSATS Institute of Information Technology, Park Road Chak Shahzad Islamabad, Pakistan

Tel.: +92 518318471

Fax: +92 518318499

E-mail: shamsul.qamar@comsats.edu.pk

S. Bibi

Department of Mathematics, Air University, PAF Complex, Sector E-9 Islamabad, Pakistan

S. Qamar, A. Seidel-Morgenstern

Max Planck Institute for Dynamics of Complex Technical Systems, Sandtorstrasse 1, 39106 Magdeburg, Germany 
interact differently with the stationary phase. Components interacting strongly with the particles transport (elute) slowly along the column as compared to the components having weaker interactions. Therefore, each component forms a concentration band profile moving with a specific velocity in the column. These velocity differences make possible, for a long enough column, to collect pure fractions of components at the outlet of the column [1-3].

The coupling of chemical or biochemical reactions and chromatographic separations leads to an integrated process for the production of high purity products. Within a chromatographic reactor the conversion of the reactants and the separation of the components takes place simultaneously. Therefore, reversible reactions can overcome the limitation of the conversion ruled by a chemical equilibrium. The reaction within integrated processes can be catalyzed either homogeneously or heterogeneously. In the case of a homogeneous catalysis the separation of the catalyst from the products has to be taken into account. Heterogeneously catalyzed reactions occur more often. In special cases, such as esterifications, the same ion exchange resin can act as catalyst for the reaction as well as adsorbent for the separation [4]. In contrast to the sequentially connected conventional reactors and separators, chromatographic reactors effectively reduce the number of units and improve the conversion, yield and separation capacity. Chromatographic reactors were studied by several authors $[1,5-23]$

Mathematical modeling and numerical analysis of chromatographic operations have received considerable attention since the late 1960s. A variety of mathematical models of various degree of complexity have been introduced in chromatography. For systems where equilibrium and mass transfer processes are represented by linear relationships, analytic solutions of the models are possible in the Laplace domain [24]. The analytical back transformations of the Laplace domain solutions are only possible in simplified situations [25]. However, numerical Laplace inversion techniques can be employed to calculate peak profiles from the analytical solution in the Laplace domain $[26,27]$. The moment generating property of the Laplace-domain solutions can be utilized to calculate moments of chromatographic peaks. Therefore, the retention time, the peak width, the number of theoretical plates, the peak asymmetry, and other chromatographic parameters of interest can be calculated using algebraic expressions. The moment analysis has been used in a number of studies of fixed-bed systems [3,24,28-33].

In this work, the semi-analytical solutions and analytical temporal moments are derived for twocomponents reactive lumped kinetic model (RLKM) considering irreversible $(A \rightarrow B)$ and reversible $(A \leftrightarrows B)$ reactions in the liquid and solid phases. Moreover, two sets of boundary conditions are considered for a rectangular pulse injection of finite width. The current work is an extension of our previous analysis for simplified models [31,32,34]. The solution procedure successively employs the Laplace transform and eigen-decomposition technique to uncouple the governing set of coupled differential equations. The resulting uncoupled systems of ODEs are solved using an elementary solution technique. For further analysis of the process, the analytical temporal moments are derived from the Laplace transformed solutions [31,35-37]. In the current situation, the analytical Laplace inversion is not possible. Therefore, the numerical Laplace inversion is applied to get back the solution in the actual time domain [26,27]. To verify the correctness of analytical results, the high-resolution finite volume scheme (HR-FVS) is applied to solve the model equations numerically [22,38]. Several case studies are carried out and analytical results are compared with those determined numerically. Good agreements in the results verify the correctness of analytical results and accuracy of the suggested numerical algorithm.

The rest of the paper is organized as follows. In Section 2, the RLKM is introduced and analytically solved for irreversible reactions. Section 3 extends this analysis to the case of reversible reaction. Section 4 presents the derivation of analytical moments for both types of reactions and two sets of boundary conditions. In Section 5, several case studies are carried out. Finally, conclusions are drawn in Section 6. 


\section{Irreversible reaction $(\mathrm{A} \rightarrow \mathrm{B})$}

A one-dimensional two-component transport model of linear reactive chromatography is considered. In this process, the component $A$ (component 1 ) converts into $B$ (component 2 ) through an first order irreversible reaction having reaction rate constants $\eta$ in the liquid phase and $\nu$ in the solid phase. The semi-analytical solutions of the model are obtained for different sets of inlet and outlet boundary conditions by means of Laplace transformation. Here, the solution procedure of Quezada et al. [35] is adopted to solve the model equations. Let $t$ represents the time coordinate and $z$ denotes the axial coordinate along the column length. Moreover, it is assumed that the axial dispersion coefficient, denoted by $D_{z}$, is same for both components. The lumped kinetic model incorporates the rate of variation of the local concentration of solute in the stationary phase. The model lumps hereby the contribution of internal and external mass transport resistances into a mass transfer coefficient. Thus, the mass balances in the liquid phase of a two-component linear RLKM can be expressed as

$$
\begin{aligned}
& \frac{\partial c_{1}}{\partial t}+u \frac{\partial c_{1}}{\partial z}=D_{z} \frac{\partial^{2} c_{1}}{\partial z^{2}}-\frac{k_{1}}{\epsilon}\left(q_{1}^{*}-q_{1}\right)-\eta_{1} c_{1}, \\
& \frac{\partial c_{2}}{\partial t}+u \frac{\partial c_{2}}{\partial z}=D_{z} \frac{\partial^{2} c_{2}}{\partial z^{2}}-\frac{k_{2}}{\epsilon}\left(q_{2}^{*}-q_{2}\right)+\eta_{1} c_{1} .
\end{aligned}
$$

For the solid phase, the governing equations are given as

$$
\begin{aligned}
& \frac{\partial q_{1}}{\partial t}=\frac{k_{1}}{1-\epsilon}\left(q_{1}^{*}-q_{1}\right)-\nu_{1} q_{1}, \\
& \frac{\partial q_{2}}{\partial t}=\frac{k_{2}}{1-\epsilon}\left(q_{2}^{*}-q_{2}\right)+\nu_{1} q_{1} .
\end{aligned}
$$

Appropriate boundary conditions at both ends of the column are also required which will be presented while deriving analytical solutions. In above equations, $c_{i}$ denotes the liquid phase concentration of $i$-th component, $q_{i}$ represents the solid phase concentration of $i$-th component, and $k_{i}$ is the mass transfer coefficient of $i$-th component. The linear adsorption isotherm for the $i$-th component equilibrium solid phase concentration is given as $q_{i}^{*}=a_{i} c_{i}, a_{i}$ represents the Henry constant, and $\epsilon \in(0,1)$ is the external porosity. Moreover, $\eta_{i}$ and $\nu_{i}$ are the $i$-th component liquid and solid phases reaction rate constants, respectively. The RLKM takes into account the mass transfer kinetics in the solid phase that makes it more accurate than the reactive equilibrium dispersive model (REDM) which assumes equilibrium in the solid phase concentration. For large values of $k_{i}(i=1,2)$, the solution of RLKM converges to that of REDM.

To simplify the notations and reduce the number of variables, the following dimensionless variables are introduced:

$$
\begin{aligned}
& x=\frac{z}{L_{\max }}, \quad \tau=\frac{u t}{L_{\max }}, \quad P e=\frac{L_{\max } u}{D_{z}}, \quad \widetilde{\eta}_{i}=\frac{L_{\max } \eta_{i}}{u}, \quad \widetilde{\nu}_{i}=\frac{L_{\max } \nu_{i}}{u}, \\
& \widetilde{k}_{i}=\frac{L_{\max } k_{i}}{u}, \quad C_{i}=\frac{c_{i}}{c_{0}}, \quad Q_{i}=\frac{q_{i}}{c_{0}}, \quad c_{0}=\max \left(c_{i, \text { inj }}\right), \quad(i=1,2),
\end{aligned}
$$

where $L_{\max }$ is the length of the column. Substituting these variables into Eqs. (1) to (4), we obtain

$$
\begin{aligned}
\frac{\partial C_{1}}{\partial \tau}+\frac{\partial C_{1}}{\partial x} & =\frac{1}{P e} \frac{\partial^{2} C_{1}}{\partial x^{2}}-\frac{\widetilde{k}_{1}}{\epsilon}\left(a_{1} C_{1}-Q_{1}\right)-\widetilde{\eta}_{1} C_{1}, \\
\frac{\partial C_{2}}{\partial \tau}+\frac{\partial C_{2}}{\partial x} & =\frac{1}{P e} \frac{\partial^{2} C_{2}}{\partial x^{2}}-\frac{\widetilde{k}_{2}}{\epsilon}\left(a_{2} C_{2}-Q_{2}\right)+\widetilde{\eta}_{1} C_{1}, \\
\frac{\partial Q_{1}}{\partial \tau} & =\frac{\widetilde{k}_{1}}{1-\epsilon}\left(a_{1} C_{1}-Q_{1}\right)-\widetilde{\nu}_{1} Q_{1},
\end{aligned}
$$




$$
\frac{\partial Q_{2}}{\partial \tau}=\frac{\widetilde{k}_{2}}{1-\epsilon}\left(a_{2} C_{2}-Q_{2}\right)+\widetilde{\nu}_{1} Q_{1} .
$$

The corresponding initial conditions in non-dimentionalize form are given as

$$
C_{i}(0, x)=\frac{c_{\mathrm{i}, \text { init }}}{c_{0}}=C_{\mathrm{i}, \text { init }}, \quad Q_{i}(0, x)=\frac{a_{i} c_{\mathrm{i}, \text { init }}}{c_{0}}=a_{i} C_{\mathrm{i}, \text { init }}, \quad(i=1,2) .
$$

After applying the Laplace transform in $\tau$ domain and eliminating the Laplace transformed solid phase concentration $\bar{Q}_{i}$ from Eqs. (6) and (7) through Eqs. (8) and (9), we obtain

$$
\begin{aligned}
& \frac{d^{2} \bar{C}_{1}}{d x^{2}}-P e \frac{d \bar{C}_{1}}{d x}-\left(r_{1}+s \alpha_{1}\right) \bar{C}_{1}=\left(-\alpha_{1}+\beta_{1}\right) C_{1, \text { init }}, \\
& \frac{d^{2} \bar{C}_{2}}{d x^{2}}-P e \frac{d \bar{C}_{2}}{d x}+r_{2} \bar{C}_{1}-s \alpha_{2} \bar{C}_{2}=-\alpha_{2} C_{2, \text { init }}-\beta_{2} C_{1, \text { init }},
\end{aligned}
$$

where

$$
\begin{aligned}
& \alpha_{1}=\operatorname{Pe}\left[1+\frac{a_{1} F \widetilde{k}_{1}\left(s+\widetilde{\nu}_{1}\right)}{s\left\{(1-\epsilon)\left(s+\widetilde{\nu}_{1}\right)+\widetilde{k}_{1}\right\}}\right], \quad \alpha_{2}=P e\left[1+\frac{a_{2} F \widetilde{k}_{2}}{s(1-\epsilon)+\widetilde{k}_{2}}\right], \quad F=\frac{1-\epsilon}{\epsilon}, \\
& r_{1}=\operatorname{Pe} \widetilde{\eta}_{1}, \quad r_{2}=\operatorname{Pe}\left[\widetilde{\eta}_{1}+\frac{a_{1} F \widetilde{\nu}_{1} \widetilde{k}_{1} \widetilde{k}_{2}}{\left\{(1-\epsilon)\left(s+\widetilde{\nu}_{1}\right)+\widetilde{k}_{1}\right\}\left\{s(1-\epsilon)+\widetilde{k}_{2}\right\}}\right], \\
& \beta_{1}=\frac{P e a_{1} F \widetilde{\nu}_{1} \widetilde{k}_{1}}{s\left\{(1-\epsilon)\left(s+\widetilde{\nu}_{1}\right)+\widetilde{k}_{1}\right\}}, \quad \beta_{2}=\frac{P e a_{1} F \widetilde{\nu}_{1} \widetilde{k}_{2}(1-\epsilon)}{\left\{(1-\epsilon)\left(s+\widetilde{\nu}_{1}\right)+\widetilde{k}_{1}\right\}\left\{s(1-\epsilon)+\widetilde{k}_{2}\right\}} .
\end{aligned}
$$

In these equations, $\bar{C}_{1}$ and $\bar{C}_{2}$ are the liquid phase concentrations of mixture components in the Laplace domain. By using matrix notation, Eqs. (11) and (12) take the following form

$$
\frac{d^{2}}{d x^{2}}\left\{\begin{array}{l}
\bar{C}_{1} \\
\bar{C}_{2}
\end{array}\right\}-P e \frac{d}{d x}\left\{\begin{array}{l}
\bar{C}_{1} \\
\bar{C}_{2}
\end{array}\right\}+\left[\begin{array}{cc}
-r_{1}-s \alpha_{1} & 0 \\
r_{2} & -s \alpha_{2}
\end{array}\right]\left\{\begin{array}{l}
\bar{C}_{1} \\
\bar{C}_{2}
\end{array}\right\}=\left\{\begin{array}{c}
\left(-\alpha_{1}+\beta_{1}\right) C_{1, \text { init }} \\
-\alpha_{2} C_{2, \text { init }}-\beta_{2} C_{1, \text { init }}
\end{array}\right\},
$$

where parenthesis [ ] stands for a square matrix and \{\} represents the column matrix. Thus, a combined reaction coefficient matrix $[B]$ on the left hand side of Eq. (14) is given as

$$
B=\left[\begin{array}{cc}
-r_{1}-s \alpha_{1} & 0 \\
r_{2} & -s \alpha_{2}
\end{array}\right] .
$$

Next, we compute the linear transformation matrix $[A]$. Note that, the columns of $[A]$ should be the eigenvectors of the combined reaction coefficient matrix $[B]$. The eigenvalues and eigenvectors of $[B]$ are given as:

$$
\lambda^{\prime}=-r_{1}-s \alpha_{1}, \quad x_{1}=\left[\begin{array}{c}
A_{11} \\
\frac{r_{2} A_{11}}{s\left(\alpha_{2}-\alpha_{1}\right)-r_{1}}
\end{array}\right] \quad \text { and } \quad \lambda^{\prime \prime}=-s \alpha_{2}, \quad x_{2}=\left[\begin{array}{c}
0 \\
A_{22}
\end{array}\right] .
$$

Here, $\lambda^{\prime}$ and $\lambda^{\prime \prime}$ are the eigenvalues and $A_{11}$ and $A_{22}$ are the arbitrary constants. For simplicity, we take the values of $A_{11}$ and $A_{22}$ equal to one. Then, using Eq. (16), the diagonal matrix $\widetilde{P}$ and the transformation matrix $[A]$ can be written as

$$
\widetilde{P}=\left[\begin{array}{cc}
-r_{1}-s \alpha_{1} & 0 \\
0 & -s \alpha_{2}
\end{array}\right], \quad A=\left[\begin{array}{cc}
1 & 0 \\
\frac{r_{2}}{s\left(\alpha_{2}-\alpha_{1}\right)-r_{1}} & 1
\end{array}\right] .
$$

The matrix $[A]$ can be used for the following linear transformation

$$
\left\{\begin{array}{l}
\bar{C}_{1} \\
\bar{C}_{2}
\end{array}\right\}=\left[\begin{array}{cc}
1 & 0 \\
\frac{r_{2}}{s\left(\alpha_{2}-\alpha_{1}\right)-r_{1}} & 1
\end{array}\right]\left\{\begin{array}{l}
b_{1} \\
b_{2}
\end{array}\right\} .
$$


By applying the above linear transformation on Eq. (14) we get

$$
\begin{aligned}
& \frac{d^{2}}{d x^{2}}\left\{\begin{array}{l}
b_{1} \\
b_{2}
\end{array}\right\}-P e \frac{d}{d x}\left\{\begin{array}{l}
b_{1} \\
b_{2}
\end{array}\right\}+\left[\begin{array}{cc}
-r_{1}-s \alpha_{1} & 0 \\
0 & -s \alpha_{2}
\end{array}\right]\left\{\begin{array}{l}
b_{1} \\
b_{2}
\end{array}\right\} \\
& =\left[\begin{array}{cc}
1 & 0 \\
\frac{-r_{2}}{s\left(\alpha_{2}-\alpha_{1}\right)-r_{1}} & 1
\end{array}\right]\left\{\begin{array}{c}
\left(-\alpha_{1}+\beta_{1}\right) C_{1, \text { init }} \\
-\alpha_{2} C_{2, \text { init }}-\beta_{2} C_{1, \text { init }}
\end{array}\right\} .
\end{aligned}
$$

One can see that Eq. (19) represents the two independent, steady state, advection-dispersion equations with decay term of first order. Next is to find the explicit solutions of these two independent ordinary differential equations

$$
\begin{aligned}
& \frac{d^{2} b_{1}}{d x^{2}}-P e \frac{d b_{1}}{d x}-\left(r_{1}+s \alpha_{1}\right) b_{1}=\left(-\alpha_{1}+\beta_{1}\right) C_{1, \text { init }}, \\
& \frac{d^{2} b_{2}}{d x^{2}}-P e \frac{d b_{2}}{d x}-s \alpha_{2} b_{2}=\frac{r_{2}\left(\alpha_{1}-\beta_{1}\right) C_{1, \text { init }}}{s\left(\alpha_{2}-\alpha_{1}\right)-r_{1}}-\alpha_{2} C_{2, \text { init }}-\beta_{2} C_{1, \text { init }} .
\end{aligned}
$$

The solutions of ODEs in Eqs. (20) and (21) are given as

$$
b_{1}(s, x)=A_{1} e^{\lambda_{1} x}+B_{1} e^{\lambda_{2} x}+\frac{\left(\alpha_{1}-\beta_{1}\right)}{r_{1}+s \alpha_{1}}, \quad \lambda_{1,2}=\frac{P e \pm \sqrt{P e^{2}+4\left(r_{1}+s \alpha_{1}\right)}}{2},
$$

and

$$
\begin{aligned}
& b_{2}(s, x)=A_{2} e^{\lambda_{3} x}+B_{2} e^{\lambda_{4} x}-\frac{1}{s \alpha_{2}}\left[\frac{r_{2}\left(\alpha_{1}-\beta_{1}\right) C_{1, \text { init }}}{s\left(\alpha_{2}-\alpha_{1}\right)-r_{1}}-\alpha_{2} C_{2, \text { init }}-\beta_{2} C_{1, \text { init }}\right], \\
& \lambda_{3,4}=\frac{P e \pm \sqrt{P e^{2}+4 s \alpha_{2}}}{2} .
\end{aligned}
$$

Here, $A_{1}, B_{1}, A_{2}$ and $B_{2}$ are integration constants which can be determined by using suitable boundary conditions (BCs) at the column inlet and outlet. In this study, we consider two sets of BCs as given below. If $r_{\mathrm{i}}=0$ (for $i=1,2$ ) and $\nu=0$, Eqs. (22) and (23) reduce to non-reactive chromatographic model equations discussed in Javeed et al. [31].

\subsection{Dirichlet BCs}

In this case, the normalized inlet BCs are given as

$$
C_{i}(\tau, 0)=\left\{\begin{array}{lc}
\frac{c_{i, \mathrm{inj}}}{c_{0}}, & \text { if } 0<\tau \leq \tau_{\mathrm{inj}}, \\
0, & \tau>\tau_{\mathrm{inj}} .
\end{array}\right.
$$

At the column outlet, the Neumann BCs for a column of hypothetically infinite length are used as

$$
\frac{\partial C_{i}}{\partial x}(\tau, \infty)=0, \quad i=1,2 .
$$

Here $c_{i, \text { inj }}$ denotes the injected concentration of component $i$ at the left end of the column (i.e. at $x=0)$. By assuming $C_{i, \text { inj }}=\frac{c_{i, \text { inj }}}{c_{0}}$ for $i=1,2$, these boundary conditions in the Laplace domain can be expressed as

$$
\bar{C}_{i}(s, 0)=\frac{C_{i, \mathrm{inj}}}{s}\left(1-e^{-s \tau_{\mathrm{inj}}}\right), \quad \frac{d \bar{C}_{i}}{d x}(s, \infty)=0, \quad i=1,2 .
$$

${ }_{45}$ Using the transformation given in Eq. (18), we get 


$$
\bar{C}_{1}(s, x)=b_{1}(s, x)
$$

$$
\begin{aligned}
\bar{C}_{2}(s, x) & =\frac{r_{2}}{s\left(\alpha_{2}-\alpha_{1}\right)-r_{1}}\left[e^{\lambda_{2} x}\left\{\frac{\left(1-e^{-s \tau_{\text {inj }}}\right)}{s} C_{1, \text { inj }}-\frac{\left(\alpha_{1}-\beta_{1}\right) C_{1, \text { init }}}{r_{1}+s \alpha_{1}}\right\}+\frac{\left(\alpha_{1}-\beta_{1}\right) C_{1, \text { init }}}{r_{1}+s \alpha_{1}}\right] \\
& +e^{\lambda_{4} x}\left[\frac{\left(1-e^{-s \tau_{\text {inj }}}\right)}{s}\left\{C_{2, \text { inj }}-\frac{r_{2} C_{1, \text { inj }}}{s\left(\alpha_{2}-\alpha_{1}\right)-r_{1}}\right\}+\frac{1}{s \alpha_{2}}\left\{\frac{\left(\alpha_{1}-P e \beta_{1}\right) r_{2} C_{1, \text { init }}}{s\left(\alpha_{2}-\alpha_{1}\right)-r_{1}}-\alpha_{2} C_{2, \text { init }}\right.\right.
\end{aligned}
$$

so that

$$
b_{2}(s, 0)=\frac{\left(1-e^{-s \tau_{\mathrm{inj}}}\right)}{s} C_{2, \mathrm{inj}}-\frac{r_{2} b_{1}(s, 0)}{s\left(\alpha_{2}-\alpha_{1}\right)-r_{1}}, \quad \frac{d b_{2}}{d x}(s, \infty)=0 .
$$

After applying the BCs in Eq. (28) on Eq. (22), the values of $A_{1}$ and $B_{1}$ are given as

$$
A_{1}=0, \quad B_{1}=\frac{\left(1-e^{-s \tau_{\mathrm{inj}}}\right)}{s} C_{1, \mathrm{inj}}-\frac{\left(\alpha_{1}-\beta_{1}\right) C_{1, \mathrm{init}}}{r_{1}+s \alpha_{1}} .
$$

By using the relation in Eq. (27), we get the Laplace domain solution as

$$
\bar{C}_{1}(s, x)=\left[\frac{\left(1-e^{-s \tau_{\text {inj }}}\right)}{s} C_{1, \text { inj }}-\frac{\left(\alpha_{1}-\beta_{1}\right) C_{1, \text { init }}}{r_{1}+s \alpha_{1}}\right] e^{\lambda_{2} x}+\frac{\left(\alpha_{1}-\beta_{1}\right) C_{1, \text { init }}}{r_{1}+s \alpha_{1}} .
$$

Similarly, using Eq. (30) in Eq. (23), we get the values of $A_{2}$ and $B_{2}$ as

$$
\begin{aligned}
A_{2}=0, \quad B_{2} & =\frac{\left(1-e^{-s \tau_{\text {inj }}}\right)}{s}\left[C_{2, \text { inj }}-\frac{r_{2} C_{1, \text { inj }}}{s\left(\alpha_{2}-\alpha_{1}\right)-r_{1}}\right] \\
& +\frac{1}{s \alpha_{2}}\left[\frac{r_{2}\left(\alpha_{1}-\beta_{1}\right) C_{1, \text { init }}}{s\left(\alpha_{2}-\alpha_{1}\right)-r_{1}}-\alpha_{2} C_{2, \text { init }}-\beta_{2} C_{1, \text { init }}\right] .
\end{aligned}
$$

Thus, Eq. (23) together with the values of $A_{2}$ and $B_{2}$ becomes

$$
\begin{aligned}
b_{2}(s, x) & =\left[\frac{\left(1-e^{-s \tau_{\text {inj }}}\right)}{s}\left\{C_{2, \text { inj }}-\frac{r_{2} C_{1, \text { inj }}}{s\left(\alpha_{2}-\alpha_{1}\right)-r_{1}}\right\}+\frac{1}{s \alpha_{2}}\left\{\frac{r_{2}\left(\alpha_{1}-\beta_{1}\right) C_{1, \text { init }}}{s\left(\alpha_{2}-\alpha_{1}\right)-r_{1}}-\alpha_{2} C_{2, \text { init }}\right.\right. \\
& \left.\left.-\beta_{2} C_{1, \text { init }}\right\}\right] e^{\lambda_{4} x}-\frac{1}{s \alpha_{2}}\left[\frac{\left(\alpha_{1}-\beta_{1}\right) r_{2} C_{1, \text { init }}}{s\left(\alpha_{2}-\alpha_{1}\right)-r_{1}}-\alpha_{2} C_{2, \text { init }}-\beta_{2} C_{1, \text { init }}\right] .
\end{aligned}
$$

After using Eq. (29) in Eq. (34), we obtain 


$$
\left.\left.-\beta_{2} C_{1, \text { init }}\right\}\right]-\frac{1}{s \alpha_{2}}\left[\frac{\left(\alpha_{1}-\beta_{1}\right) r_{2} C_{1, \text { init }}}{s\left(\alpha_{2}-\alpha_{1}\right)-r_{1}}-\alpha_{2} C_{2, \text { init }}-\beta_{2} C_{1, \text { init }}\right] .
$$

The solutions in $\tau$ domain $C_{i}(\tau, x)$ can be achieved by using the following expression for the back transformation:

$$
C_{j}(\tau, x)=\frac{1}{2 \pi i} \int_{\gamma-i \infty}^{\gamma+i \infty} e^{-\tau s} \bar{C}_{j}(s, x) d s, \quad j=1,2,
$$

where, $\gamma$ is a real constant that exceeds the real part of all the singularities of $\bar{C}_{j}(s, x)$.

In this case, no analytical Laplace inversion is possible. Therefore, numerical Laplace inversion is adopted to get back solutions in the actual time domain $[26,27,31,32]$. In this technique, the exact integrals of back transformation (c.f. Eq. (36)) are approximated by using Fourier series [27].

\subsection{Danckwerts boundary conditions}

In this case, the finite length column is considered. The normalized boundary conditions have the form [39]

$$
C_{i}(\tau, 0)=\left\{\begin{array}{l}
C_{i, \text { inj }}+\frac{1}{P e} \frac{\partial C_{i}}{\partial x}(\tau, 0), \quad \text { if } 0<\tau \leq \tau_{\text {inj }}, \quad i=1,2, \\
0, \quad \tau>\tau_{\text {inj }},
\end{array}\right.
$$

and

$$
\frac{\partial C_{i}}{\partial x}(\tau, 1)=0, \quad i=1,2 .
$$

Using the Laplace transformation, we get

$$
\bar{C}_{i}(s, 0)=\frac{\left(1-e^{-s \tau_{\mathrm{inj}}}\right)}{s} C_{i, \mathrm{inj}}+\frac{1}{P e} \frac{d \bar{C}_{i}}{d x}(s, 0), \quad \frac{d \bar{C}_{i}}{d x}(s, 1)=0 .
$$

Following the same solution procedure as discussed in Subsection 2.1, the solutions in the Laplace domain are given as

$$
\begin{aligned}
\bar{C}_{1}(s, x) & =\left[\frac{\lambda_{2} e^{\lambda_{2}+\lambda_{1} x}-\lambda_{1} e^{\lambda_{1}+\lambda_{2} x}}{\left(1-\frac{\lambda_{1}}{P e}\right) \lambda_{2} e^{\lambda_{2}}-\left(1-\frac{\lambda_{2}}{P e}\right) \lambda_{1} e^{\lambda_{1}}}\right]\left[\frac{\left(1-e^{-s \tau_{\mathrm{inj}}}\right)}{s} C_{1, \text { inj }}-\frac{\left(\alpha_{1}-\beta_{1}\right) C_{1, \text { init }}}{r_{1}+s \alpha_{1}}\right] \\
& +\frac{\left(\alpha_{1}-\beta_{1}\right) C_{1, \text { init }}}{r_{1}+s \alpha_{1}}
\end{aligned}
$$

and

$$
\begin{aligned}
\bar{C}_{2}(s, x) & =\left[\frac{r_{2}}{s\left(\alpha_{2}-\alpha_{1}\right)-r_{1}}\right]\left[\frac{\lambda_{2} e^{\lambda_{2}+\lambda_{1} x}-\lambda_{1} e^{\lambda_{1}+\lambda_{2} x}}{\left(1-\frac{\lambda_{1}}{P e}\right) \lambda_{2} e^{\lambda_{2}}-\left(1-\frac{\lambda_{2}}{P e}\right) \lambda_{1} e^{\lambda_{1}}}\right]\left[\frac{\left(1-e^{-s \tau_{\mathrm{inj}}}\right)}{s} C_{1, \mathrm{inj}}\right. \\
& \left.-\frac{\left(\alpha_{1}-\beta_{1}\right) C_{1, \mathrm{init}}}{r_{1}+s \alpha_{1}}\right]+\frac{\left(\alpha_{1}-\beta_{1}\right) C_{1, \mathrm{init}}}{r_{1}+s \alpha_{1}}\left[\frac{r_{2}}{s\left(\alpha_{2}-\alpha_{1}\right)-r_{1}}\right] \\
& +\left[\frac{\lambda_{4} e^{\lambda_{4}+\lambda_{3} x}-\lambda_{3} e^{\lambda_{3}+\lambda_{4} x}}{\left(1-\frac{\lambda_{3}}{P e}\right) \lambda_{4} e^{\lambda_{4}}-\left(1-\frac{\lambda_{4}}{P e}\right) \lambda_{3} e^{\lambda_{3}}}\right]\left[\left(\frac{1-e^{-s \tau_{\mathrm{inj}}}}{s}\right)\left\{C_{2, \mathrm{inj}}-\frac{r_{2} C_{1, \mathrm{inj}}}{s\left(\alpha_{2}-\alpha_{1}\right)-r_{1}}\right\}\right.
\end{aligned}
$$




$$
\begin{aligned}
& \left.+\frac{1}{s \alpha_{2}}\left\{\frac{\left(\alpha_{1}-\beta_{1}\right) r_{2} C_{1, \text { init }}}{s\left(\alpha_{2}-\alpha_{1}\right)-r_{1}}-\alpha_{2} C_{2, \text { init }}-\beta_{2} C_{1, \text { init }}\right\}\right] \\
& -\frac{1}{s \alpha_{2}}\left[\frac{\left(\alpha_{1}-\beta_{1}\right) r_{2} C_{1, \text { init }}}{s\left(\alpha_{2}-\alpha_{1}\right)-r_{1}}-\alpha_{2} C_{2, \text { init }}-\beta_{2} C_{1, \text { init }}\right] .
\end{aligned}
$$

Once again, no analytical Laplace inversion is possible. Therefore, the numerical Laplace inversion is applied to get back solutions in the actual time domain $[26,27,31,32]$.

\section{Reversible reaction $A \rightleftharpoons B$}

In this section, the more general case of linear reaction, i.e. reversible reaction, is presented. For the considered case, the injected component $A$ (component 1 ) converts to the component $B$ (component 2) with reaction rate constants $\eta_{1}$ in the liquid phase and $\nu_{1}$ in the solid phase. Because of the reversible reaction, component $B$ is converted partly back to component $A$ with a reaction rate constant $\eta_{2}$ in liquid phase and $\nu_{2}$ in the solid phase. Thus, the governing equations of two-components RLKM in the liquid phase are formulated as

$$
\begin{aligned}
& \frac{\partial c_{1}}{\partial t}+u \frac{\partial c_{1}}{\partial z}=D \frac{\partial^{2} c_{1}}{\partial z^{2}}-\frac{k_{1}}{\epsilon}\left(q_{1}^{*}-q_{1}\right)-\eta_{1} c_{1}+\eta_{2} c_{2}, \\
& \frac{\partial c_{2}}{\partial t}+u \frac{\partial c_{2}}{\partial z}=D \frac{\partial^{2} c_{2}}{\partial z^{2}}-\frac{k_{2}}{\epsilon}\left(q_{2}^{*}-q_{2}\right)+\eta_{1} c_{1}-\eta_{2} c_{2} .
\end{aligned}
$$

For the solid phase, the governing equations are

$$
\begin{aligned}
& \frac{\partial q_{1}}{\partial t}=\frac{k_{1}}{1-\epsilon}\left(q_{1}^{*}-q_{1}\right)-\nu_{1} q_{1}+\nu_{2} q_{2}, \\
& \frac{\partial q_{2}}{\partial t}=\frac{k_{2}}{1-\epsilon}\left(q_{2}^{*}-q_{2}\right)+\nu_{1} q_{1}-\nu_{2} q_{2} .
\end{aligned}
$$

Using dimensionless variables given in Eq. (5) and linear adsorption isotherms, the above equations in normalized form can be rewritten as

$$
\begin{aligned}
& \frac{\partial C_{1}}{\partial \tau}+\frac{\partial C_{1}}{\partial x}=\frac{1}{P e} \frac{\partial^{2} C_{1}}{\partial x^{2}}-\frac{\widetilde{k}_{1}}{\epsilon}\left(a_{1} C_{1}-Q_{1}\right)-\widetilde{\eta}_{1} C_{1}+\widetilde{\eta}_{2} C_{2}, \\
& \frac{\partial C_{2}}{\partial \tau}+\frac{\partial C_{2}}{\partial x}=\frac{1}{P e} \frac{\partial^{2} C_{2}}{\partial x^{2}}-\frac{\widetilde{k}_{2}}{\epsilon}\left(a_{2} C_{2}-Q_{2}\right)+\widetilde{\eta}_{1} C_{1}-\widetilde{\eta}_{2} C_{2}, \\
& \frac{\partial Q_{1}}{\partial \tau}=\frac{\widetilde{k}_{1}}{1-\epsilon}\left(a_{1} C_{1}-Q_{1}\right)-\widetilde{\nu}_{1} Q_{1}+\widetilde{\nu}_{2} Q_{2}, \\
& \frac{\partial Q_{2}}{\partial \tau}=\frac{\widetilde{k}_{2}}{1-\epsilon}\left(a_{2} C_{2}-Q_{2}\right)+\widetilde{\nu}_{1} Q_{1}-\widetilde{\nu}_{2} Q_{2} .
\end{aligned}
$$

By applying the Laplace transformation in $\tau$ domain and eliminating Eqs. (48) and (49), Eqs. (46) and (47) take the forms

$$
\begin{aligned}
& \frac{d^{2} \bar{C}_{1}}{d x^{2}}-P e \frac{d \bar{C}_{1}}{d x}-\left(r_{1}+s \alpha_{1}\right) \bar{C}_{1}+r_{2} \bar{C}_{2}=-\beta_{1} C_{1, \text { init }}-\beta_{2} C_{2, \text { init }}, \\
& \frac{d^{2} \bar{C}_{2}}{d x^{2}}-P e \frac{d \bar{C}_{2}}{d x}+r_{3} \bar{C}_{1}-\left(r_{4}+s \alpha_{2}\right) \bar{C}_{2}=-\beta_{3} C_{1, \text { init }}-\beta_{4} C_{2, \text { init }},
\end{aligned}
$$

where 


$$
\begin{aligned}
& \alpha_{1}=P e\left[1+\frac{a_{1} F \widetilde{k}_{1}}{(1-\epsilon)\left(s+\widetilde{\nu}_{1}\right)+\widetilde{k}_{1}}+\frac{a_{1} F \widetilde{\nu}_{1} \widetilde{k}_{1}}{s\left\{\left(s+\widetilde{\nu}_{1}\right)(1-\epsilon)+\widetilde{k}_{1}\right\}}\right. \\
& \left.-\frac{a_{1} F \widetilde{\nu}_{1} \widetilde{\nu}_{2} \widetilde{k}_{1}^{2}(1-\epsilon)}{s\left\{\left(s+\widetilde{\nu}_{1}\right)(1-\epsilon)+\widetilde{k}_{1}\right\}\left[\left\{\left(s+\widetilde{\nu}_{1}\right)(1-\epsilon)+\widetilde{k}_{1}\right\}\left\{\left(s+\widetilde{\nu}_{2}\right)(1-\epsilon)+\widetilde{k}_{2}\right\}-\widetilde{\nu}_{1} \widetilde{\nu}_{2}(1-\epsilon)^{2}\right]}\right] \text {, } \\
& \alpha_{2}=P e\left[1+\frac{a_{2} \widetilde{k}_{2}}{s \epsilon}-\frac{a_{2} \widetilde{k}_{2}^{2}\left\{\left(s+\widetilde{\nu}_{1}\right)(1-\epsilon)+\widetilde{k}_{1}\right\}}{s \epsilon\left[\left\{\left(s+\widetilde{\nu}_{1}\right)(1-\epsilon)+\widetilde{k}_{1}\right\}\left\{\left(s+\widetilde{\nu}_{2}\right)(1-\epsilon)+\widetilde{k}_{2}\right\}-\widetilde{\nu}_{1} \widetilde{\nu}_{2}(1-\epsilon)^{2}\right]}\right] \text {, } \\
& F=\frac{1-\epsilon}{\epsilon}, \quad r_{1}=P e \widetilde{\eta}_{1}, \quad r_{4}=P e \widetilde{\eta}_{2}, \\
& r_{2}=P e\left[\widetilde{\eta}_{2}+\frac{a_{2} F \widetilde{\nu}_{2} \widetilde{k}_{1} \widetilde{k}_{2}}{\left\{\left(s+\widetilde{\nu}_{1}\right)(1-\epsilon)+\widetilde{k}_{1}\right\}\left\{\left(s+\widetilde{\nu}_{2}\right)(1-\epsilon)+\widetilde{k}_{2}\right\}-\widetilde{\nu}_{1} \widetilde{\nu}_{2}(1-\epsilon)^{2}}\right], \\
& r_{3}=P e\left[\widetilde{\eta}_{1}+\frac{a_{1} F \widetilde{\nu}_{1} \widetilde{k}_{1} \widetilde{k}_{2}}{\left\{\left(s+\widetilde{\nu}_{1}\right)(1-\epsilon)+\widetilde{k}_{1}\right\}\left\{\left(s+\widetilde{\nu}_{2}\right)(1-\epsilon)+\widetilde{k}_{2}\right\}-\widetilde{\nu}_{1} \widetilde{\nu}_{2}(1-\epsilon)^{2}}\right] \text {, } \\
& \beta_{1}=P e\left[1+\frac{a_{1} F \widetilde{k}_{1}}{\left(s+\widetilde{\nu}_{1}\right)(1-\epsilon)+\widetilde{k}_{1}}\right. \\
& \left.+\frac{a_{1} F \widetilde{\nu}_{1} \widetilde{\nu}_{2} \widetilde{k}_{1}(1-\epsilon)^{2}}{\left\{\left(s+\widetilde{\nu}_{1}\right)(1-\epsilon)+\widetilde{k}_{1}\right\}\left[\left\{\left(s+\widetilde{\nu}_{1}\right)(1-\epsilon)+\widetilde{k}_{1}\right\}\left\{\left(s+\widetilde{\nu}_{2}\right)(1-\epsilon)+\widetilde{k}_{2}\right\}-\widetilde{\nu}_{1} \widetilde{\nu}_{2}(1-\epsilon)^{2}\right]}\right] \text {, } \\
& \beta_{2}=\frac{P e a_{2} F \widetilde{\nu}_{2} \widetilde{k}_{1}(1-\epsilon)}{\left\{\left(s+\widetilde{\nu}_{1}\right)(1-\epsilon)+\widetilde{k}_{1}\right\}\left\{\left(s+\widetilde{\nu}_{2}\right)(1-\epsilon)+\widetilde{k}_{2}\right\}-\widetilde{\nu}_{1} \widetilde{\nu}_{2}(1-\epsilon)^{2}}, \\
& \beta_{3}=\frac{P e a_{1} F \widetilde{\nu}_{1} \widetilde{k}_{2}(1-\epsilon)}{\left\{\left(s+\widetilde{\nu}_{1}\right)(1-\epsilon)+\widetilde{k}_{1}\right\}\left\{\left(s+\widetilde{\nu}_{2}\right)(1-\epsilon)+\widetilde{k}_{2}\right\}-\widetilde{\nu}_{1} \widetilde{\nu}_{2}(1-\epsilon)^{2}}, \\
& \beta_{4}=P e\left[1+\frac{a_{2} F \widetilde{k}_{2}\left\{\left(s+\widetilde{\nu}_{1}\right)(1-\epsilon)+\widetilde{k}_{1}\right\}}{\left\{\left(s+\widetilde{\nu}_{1}\right)(1-\epsilon)+\widetilde{k}_{1}\right\}\left\{\left(s+\widetilde{\nu}_{2}\right)(1-\epsilon)+\widetilde{k}_{2}\right\}-\widetilde{\nu}_{1} \widetilde{\nu}_{2}(1-\epsilon)^{2}}\right] .
\end{aligned}
$$

The two sets of BCs introduced in Section 2 are considered again. Adopting the same solution procedure as discussed in the previous Section, we obtain the following solutions.

\subsection{Dirichlet boundary conditions}

In this case, the boundary conditions in Eqs. (24) and (25) are taken into account. Thus, the Laplace domain solutions are given as

$$
\begin{aligned}
\bar{C}_{1}(s, x) & =e^{m_{2} x}\left[\frac{\lambda_{3}\left(1-e^{-s \tau_{\mathrm{inj}}}\right)\left(r_{3} C_{1, \mathrm{inj}}-\lambda_{4} C_{2, \mathrm{inj}}\right)}{s r_{3}\left(\lambda_{3}-\lambda_{4}\right)}\right. \\
& \left.+\frac{\lambda_{3}\left\{\left(\beta_{1} r_{3}-\beta_{3} \lambda_{4}\right) C_{1, \mathrm{init}}+\left(\beta_{2} r_{3}-\beta_{4} \lambda_{4}\right) C_{2, \text { init }}\right\}}{\lambda_{1} r_{3}\left(\lambda_{3}-\lambda_{4}\right)}\right] \\
& -e^{m_{4} x}\left[\frac{\lambda_{4}\left(1-e^{-s \tau_{\mathrm{inj}}}\right)\left(r_{3} C_{1, \mathrm{inj}}-\lambda_{3} C_{2, \mathrm{inj}}\right)}{s r_{3}\left(\lambda_{3}-\lambda_{4}\right)}\right. \\
& \left.-\frac{\lambda_{4}\left\{\left(-\beta_{1} r_{3}+\beta_{3} \lambda_{3}\right) C_{1, \text { init }}+\left(-\beta_{2} r_{3}+\beta_{4} \lambda_{3}\right) C_{2, \text { init }}\right\}}{\lambda_{2} r_{3}\left(\lambda_{3}-\lambda_{4}\right)}\right] \\
& -\frac{C_{1, \text { init }}\left\{\beta_{1} r_{3}\left(\lambda_{2} \lambda_{3}-\lambda_{1} \lambda_{4}\right)-\beta_{3} \lambda_{3} \lambda_{4}\left(\lambda_{2}-\lambda_{1}\right)\right\}}{\lambda_{1} \lambda_{2} r_{3}\left(\lambda_{3}-\lambda_{4}\right)}
\end{aligned}
$$




$$
\begin{aligned}
+ & \frac{C_{2, \text { init }}\left\{\beta_{4} \lambda_{3} \lambda_{4}\left(\lambda_{2}-\lambda_{1}\right)-\beta_{2} r_{3}\left(\lambda_{2} \lambda_{3}-\lambda_{1} \lambda_{4}\right)\right\}}{\lambda_{1} \lambda_{2} r_{3}\left(\lambda_{3}-\lambda_{4}\right)}, \\
\bar{C}_{2}(s, x) & =e^{m_{2} x}\left[\frac{\left(1-e^{-s \tau_{\text {inj }}}\right)\left(r_{3} C_{1, \text { inj }}-\lambda_{4} C_{2, \text { inj }}\right)}{s\left(\lambda_{3}-\lambda_{4}\right)}\right. \\
& \left.+\frac{\left\{\left(\beta_{1} r_{3}-\beta_{3} \lambda_{4}\right) C_{1, \text { init }}+\left(\beta_{2} r_{3}-\beta_{4} \lambda_{4}\right) C_{2, \text { init }}\right\}}{\lambda_{1}\left(\lambda_{3}-\lambda_{4}\right)}\right] \\
& -e^{m_{4} x}\left[\frac{\left(1-e^{-s \tau_{\text {inj }}}\right)\left(r_{3} C_{1, \text { inj }}-\lambda_{3} C_{2, \text { inj }}\right)}{s\left(\lambda_{3}-\lambda_{4}\right)}\right. \\
& \left.-\frac{\left\{\left(-\beta_{1} r_{3}+\beta_{3} \lambda_{3}\right) c_{1, \text { init }}+\left(-\beta_{2} r_{3}+\beta_{4} \lambda_{3}\right) C_{2, \text { init }}\right\}}{\lambda_{2}\left(\lambda_{3}-\lambda_{4}\right)}\right] \\
& -\frac{C_{1, \text { init }}\left\{\beta_{1} r_{3}\left(\lambda_{2}-\lambda_{1}\right)+\beta_{3}\left(\lambda_{1} \lambda_{3}-\lambda_{2} \lambda_{4}\right)\right\}}{\lambda_{1} \lambda_{2}\left(\lambda_{3}-\lambda_{4}\right)} \\
& +\frac{C_{2, \text { init }}\left\{\beta_{4}\left(\lambda_{2} \lambda_{4}-\lambda_{1} \lambda_{3}\right)-\beta_{2} r_{3}\left(\lambda_{2}-\lambda_{1}\right)\right\}}{\lambda_{1} \lambda_{2}\left(\lambda_{3}-\lambda_{4}\right)},
\end{aligned}
$$

$$
\begin{aligned}
\bar{C}_{1}(s, x) & =\frac{\left(1-e^{-s \tau_{\mathrm{inj}}}\right) \lambda_{3}\left(r_{3} C_{1, \mathrm{inj}}-\lambda_{4} C_{2, \mathrm{inj}}\right)\left(m_{1} e^{m_{1}+m_{2} x}-m_{2} e^{m_{2}+m_{1} x}\right)}{r_{3} s\left(\lambda_{3}-\lambda_{4}\right)\left\{\left(1-\frac{m_{2}}{P e}\right) m_{1} e^{m_{1}}-\left(1-\frac{m_{1}}{P e}\right) m_{2} e^{m_{2}}\right\}} \\
& -\frac{\left(1-e^{-s \tau_{\mathrm{inj}}}\right) \lambda_{4}\left(r_{3} C_{1, \mathrm{inj}}-\lambda_{3} C_{2, \mathrm{inj}}\right)\left(m_{3} e^{m_{3}+m_{4} x}-m_{4} e^{m_{4}+m_{3} x}\right)}{r_{3} s\left(\lambda_{3}-\lambda_{4}\right)\left\{\left(1-\frac{m_{4}}{P e}\right) m_{3} e^{m_{3}}-\left(1-\frac{m_{3}}{P e}\right) m_{4} e^{m_{4}}\right\}} \\
& +\frac{\lambda_{3}\left\{\left(\beta_{1} r_{3}-\beta_{3} \lambda_{4}\right) C_{1, \text { init }}-\left(\beta_{4} \lambda_{4}-\beta_{2} r_{3}\right) C_{2, \text { init }}\right\}\left(m_{1} e^{m_{1}+m_{2} x}-m_{2} e^{m_{2}+m_{1} x}\right)}{r_{3} \lambda_{1}\left(\lambda_{3}-\lambda_{4}\right)\left\{\left(1-\frac{m_{2}}{P e}\right) m_{1} e^{m_{1}}-\left(1-\frac{m_{1}}{P e}\right) m_{2} e^{m_{2}}\right\}} \\
& -\frac{\lambda_{4}\left\{\left(\beta_{1} r_{3}-\beta_{3} \lambda_{3}\right) C_{1, \text { init }}-\left(\beta_{4} \lambda_{3}-\beta_{2} r_{3}\right) C_{2, \text { init }}\right\}\left(m_{3} e^{m_{3}+m_{4} x}-m_{4} e^{m_{4}+m_{3} x}\right)}{r_{3} \lambda_{2}\left(\lambda_{3}-\lambda_{4}\right)\left\{\left(1-\frac{m_{4}}{P e}\right) m_{3} e^{m_{3}}-\left(1-\frac{m_{3}}{P e}\right) m_{4} e^{m_{4}}\right\}} \\
& -\frac{C_{1, \text { init }}\left\{\beta_{1} r_{3}\left(\lambda_{2} \lambda_{3}-\lambda_{1} \lambda_{4}\right)-\beta_{3} \lambda_{3} \lambda_{4}\left(\lambda_{2}-\lambda_{1}\right)\right\}}{\lambda_{1} \lambda_{2} r_{3}\left(\lambda_{3}-\lambda_{4}\right)} \\
& +\frac{C_{2, \text { init }}\left\{\beta_{4} \lambda_{3} \lambda_{4}\left(\lambda_{2}-\lambda_{1}\right)-\beta_{2} r_{3}\left(\lambda_{2} \lambda_{3}-\lambda_{1} \lambda_{4}\right)\right\}}{\lambda_{1} \lambda_{2} r_{3}\left(\lambda_{3}-\lambda_{4}\right)},
\end{aligned}
$$

196 and 


$$
\begin{aligned}
\bar{C}_{2}(s, x) & =\frac{\left(1-e^{-s \tau_{\text {inj }}}\right)\left(r_{3} C_{1, \text { inj }}-\lambda_{4} C_{2, \text { inj }}\right)\left(m_{1} e^{m_{1}+m_{2} x}-m_{2} e^{m_{2}+m_{1} x}\right)}{s\left(\lambda_{3}-\lambda_{4}\right)\left\{\left(1-\frac{m_{2}}{P e}\right) m_{1} e^{m_{1}}-\left(1-\frac{m_{1}}{P e}\right) m_{2} e^{m_{2}}\right\}} \\
& -\frac{\left(1-e^{-s \tau_{\text {inj }}}\right)\left(r_{3} C_{1, \text { inj }}-\lambda_{3} C_{2, \text { inj }}\right)\left(m_{3} e^{m_{3}+m_{4} x}-m_{4} e^{m_{4}+m_{3} x}\right)}{s\left(\lambda_{3}-\lambda_{4}\right)\left\{\left(1-\frac{m_{4}}{P e}\right) m_{3} e^{m_{3}}-\left(1-\frac{m_{3}}{P e}\right) m_{4} e^{m_{4}}\right\}} \\
& +\frac{\left\{\left(\beta_{1} r_{3}-\beta_{3} \lambda_{4}\right) c_{1, \text { init }}-\left(\beta_{4} \lambda_{4}-\beta_{2} r_{3}\right) C_{2, \text { init }}\right\}\left(m_{1} e^{m_{1}+m_{2} x}-m_{2} e^{m_{2}+m_{1} x}\right)}{\lambda_{1}\left(\lambda_{3}-\lambda_{4}\right)\left\{\left(1-\frac{m_{2}}{P e}\right) m_{1} e^{m_{1}}-\left(1-\frac{m_{1}}{P e}\right) m_{2} e^{m_{2}}\right\}} \\
& -\frac{\left\{\left(\beta_{1} r_{3}-\beta_{3} \lambda_{3}\right) c_{1, \text { init }}-\left(\beta_{4} \lambda_{3}-\beta_{2} r_{3}\right) C_{2, \text { init }}\right\}\left(m_{3} e^{m_{3}+m_{4} x}-m_{4} e^{m_{4}+m_{3} x}\right)}{\lambda_{2}\left(\lambda_{3}-\lambda_{4}\right)\left\{\left(1-\frac{m_{4}}{P e}\right) m_{3} e^{m_{3}}-\left(1-\frac{m_{3}}{P e}\right) m_{4} e^{m_{4}}\right\}} \\
& -\frac{C_{1, \text { init }}\left\{\beta_{1} r_{3}\left(\lambda_{2}-\lambda_{1}\right)+\beta_{3}\left(\lambda_{1} \lambda_{3}-\lambda_{2} \lambda_{4}\right)\right\}}{\lambda_{1} \lambda_{2}\left(\lambda_{3}-\lambda_{4}\right)} \\
& +\frac{C_{2, \text { init }}\left\{\beta_{4}\left(\lambda_{2} \lambda_{4}-\lambda_{1} \lambda_{3}\right)-\beta_{2} r_{3}\left(\lambda_{2}-\lambda_{1}\right)\right\}}{\lambda_{1} \lambda_{2}\left(\lambda_{3}-\lambda_{4}\right)} .
\end{aligned}
$$

Here, $\lambda_{i}$ and $m_{i}$ for $i=1,2,3,4$ are given by Eqs. (55), (56) and (57). Again, the numerical Laplace inversion is applied to get the actual time domain solutions $[26,27,31,32]$.

The above models and their derived analytical solutions are considered as very flexible and versatile, both in terms of incorporated mass transfer mechanisms and regarding the option that reactions can take place independently in both phases. Many possible reactions can be treated in a simplified manner using these models. They are particularly applicable to isomerization reactions. Additionally, the important class of enantiomerization reactions, which are desired (or needed) to be suppressed in chromatographic columns, can be analyzed [41,42].

\section{Moments analysis}

Moment analysis is an attractive technique for deducing important information about the retention equilibrium and mass transfer kinetics in the column. Such a moment analysis approach has been found instructive in the literature $[3,24,31,33]$. A set of statistical temporal moments can define the appearance of the plotted elution profile. For example, the appropriate forms of the first, second and third moments can describe the mean, spread and skew of the distribution, respectively,.

The Laplace domain solutions can be utilized to obtain moments. The retention equilibriumconstant and parameters of the mass transfer kinetics in the column are related to the moments. A comparison of theoretical and experiential moments can help to estimate dispersion and other mass transfer coefficients.

In order to calculate analytical moments, the following moment generating properties of the Laplace domain solutions are exploited [31]:

The zeroth moments are defined as

$$
\mu_{0}^{(i)}=\lim _{s \rightarrow 0}\left(\bar{C}_{i}(s, x=1)\right), \quad i=1,2,
$$

and the $n$-th moments are given as

$$
\mu_{n}^{(i)}=(-1)^{n} \frac{1}{\mu_{0}^{(i)}} \lim _{s \rightarrow 0} \frac{d^{n}\left(\bar{C}_{i}(s, x=1)\right)}{d s^{n}}, \quad n=1,2,3, \cdots
$$

In this work, the central moments up to third order are derived for both sets of BCs. A complete derivation of these moments is presented in the appendix A considering a regenerated system, i.e. $c_{i, \text { init }}=0($ for $i=1,2)$, and only a solid phase reaction, i.e. $\eta_{i}=0$. 
In parallel, we integrate the concentration profiles in $\tau$ domain to compute the numerical moments. The normalized $n$-th temporal moments of the band profiles at the outlet of chromatographic column of length $x=1$ are defined as

$$
\mu_{n}^{(i)}=\frac{\int_{0}^{\infty} C_{i}(\tau, x=1) \tau^{n} d \tau}{\int_{0}^{\infty} C_{i}(\tau, x=1) d \tau}, \quad n=0,1,2, \cdots, \quad i=1,2 .
$$

While, the $n$-th central moments are expressed as

$$
{\mu^{\prime}}_{n}^{(i)}=\frac{\int_{0}^{\infty} C_{i}(\tau, x=1)\left(\tau-\mu_{1}^{(i)}\right)^{n} d \tau}{\int_{0}^{\infty} C(\tau, x=1) d \tau}, \quad n=0,1,2, \cdots, \quad i=1,2 .
$$

Below in the discussion of the test problems, a comparison of analytically and numerically determined temporal moments will be presented. The numerical moments were obtained by integrating concentration profiles generated by the high resolution FVS (c.f. Eqs. (62) and (63) ) [38]. The analytical expressions of $\mu_{2}^{\prime(i)}$ and $\mu_{3}^{\prime(i)}$ were very lengthy in some cases, therefore, only plots of these central moments are presented. The trapezoidal rule is applied to numerically approximate the integral terms appearing in these equations.

\section{Numerical test problems}

In this section, several test problems are presented to verify the correctness and usage of derived analytical results for practical problems. For that purpose, the derived semi-analytical solutions of two-components RLKM are compared with the numerical solutions of a HR-FVS $[38,40]$. The basic parameters used in the selected test problems are listed in Table 1.

\subsection{Irreversible reaction}

Figure 1 shows the outlet concentration profiles after injecting a pulse of finite width in an empty column $\left(c_{i, \text { init }}=0 g L^{-1}\right.$ for $\left.i=1,2\right)$ considering Dirichlet BCs (c.f. Eqs. (24) and (25)) and irreversible reaction. Here, the semi-analytical solution and the numerical solutions of HR-FVS are compared. Both liquid and solid phases reactions are considered. In Figure 1a, only component 1 is injected (i.e. $c_{1, \text { inj }}=1.0 \mathrm{gL}^{-1}, c_{2, \text { inj }}=0.0 \mathrm{gL}^{-1}$ ) and the liquid phase reaction is assumed to be zero, while in Figure 1b both components are injected (i.e. $c_{1, \text { inj }}=1.0 \mathrm{gL}^{-1}$ and $c_{2, \text { inj }}=0.5 \mathrm{gL}^{-1}$ ) and neglecting aging the liquid phase reaction. Both plots show that reactant (component 1), which has larger adsorption equilibrium constant, elutes later from the column as compared to the product (component 2). On the other hand, plots in Figures 1c \& 1d show the outlet concentration profiles when reactions in both solid and liquid phases are considered. It can be observed from the plots that the amount of component 2 further increases when the effects of both solid and liquid phases reactions are considered. A good agreement between the semi-analytical and numerical solutions verify the correctness of semi-analytical solutions and accuracy of the numerical solution technique.

Figure 2 depicts the effects of rate constants on the elution profiles at the column outlet considering Dirichlet BCs, $c_{i, \text { init }}=0.0 g L^{-1}$ (for $i=1,2$ ), $c_{1, \text { inj }}=1.0 g L^{-1}$, and $c_{2, \text { inj }}=0.0 g L^{-1}$. Figure 2a displays the elution profiles for different values of the solid phase reaction rate constant, while keeping the liquid phase reaction rate fixed $\left(\widetilde{\eta}=0.42\right.$ or $\left.\eta=0.05 \mathrm{~min}^{-1}\right)$. On the other hand, Figure $2 \mathrm{~b}$ shows the elution profiles for different values of liquid phase reaction rate constant, while keeping the solid phase reaction rate constant fixed $\left(\widetilde{\nu}=0.25\right.$ or $\left.\nu=0.03 \mathrm{~min}^{-1}\right)$. Both plots reveal that the amount of product, i.e. component 2, is increased by increasing the values of reaction rate constants. Figure 3a compares the solutions for Dirichlet and Danckwerts BCs considering both solid and liquid phases reactions and two different Peclet numbers $(\mathrm{Pe})$. Here, 
we have chosen $c_{1, \text { inj }}=1.0 \mathrm{gL}^{-1}, c_{2, \text { inj }}=0.0 \mathrm{gL} L^{-1}, c_{i \text {,init }}=0.0 \mathrm{gL} L^{-1}$ (for $i=1,2$ ), and other parameters are given in Table 1. It can be observed that for smaller $P e$ numbers, i.e. larger axial dispersion coefficients, the effect of BCs are effective. Thus, Danckwerts BCs, which account for the back mixing, should be used in the case of large dispersion coefficient (i.e. small $P e$ ). Figure $3 \mathrm{~b}$ shows that the solution profiles become sharper for large values of the mass transfer coefficients $k_{i}$ (for $i=1,2$ ) and become identical to solution profiles in [34]. On the other hand, the solution profiles are spreading for small values of $k_{i}$.

A quantitative comparison of moments obtained analytically, through numerical Laplace inversion, and through HR-FVS is presented in Figure 4 for a wide range of flow rates considering Dirichlet BCs, $\eta_{1}=0 \mathrm{~min}^{-1}, \nu_{1}=0.03 \mathrm{~min}^{-1}, c_{i, \text { init }}=0 \mathrm{gL}^{-1}($ for $i=1,2), c_{1, \text { inj }}=1 \mathrm{~g} / \mathrm{l}$, and $c_{2, \text { inj }}=$ $0 g L^{-1}$. To write the moments in Appendix A with dimensions, we multiplied the zeroth moments $\mu_{0}^{(i)}$ with $L_{\max } / u$ and the $n$-th moments $\mu_{n}^{(i)}$ with $\left(L_{\max } / u\right)^{n}$ (for $\left.n=1,2, \cdots\right)$. The zeroth, first, second, and third moments are plotted versus $u, 1 / u, 1 / u^{3}$, and $1 / u^{5}$ of the derived solutions. The zeroth moment $\mu_{0}^{(i)}$ (for $i=1,2$ ), represent total mass of the solutes. The zeroth moments are shown in Figure 4a. The first moment, $\mu_{1}^{(i)}$ for $i=1,2$, reveal the expected linear trend of the mean retention time over $1 / u$, see Figure $4 \mathrm{~b}$. Figure $4 \mathrm{c}$ displays the second central moment that quantify the variance of the solution profiles. The third central moment, $\mu_{3}^{\prime(i)}$ (for $i=1,2$ ), describing the skewness of elution profiles, is shown in Figure 4d.

Good agreements in the results demonstrate the correctness of analytical calculations and the accuracy HR-FVS. The plots for Dankwerts BCs have similar behavior and are therefore omitted. One can see that due to mass transfer coefficient considered in the RLKM, the plots of second and third central moments are little bit different from those presented in Qamar et al. [34]. However, the general trend of the moments plots is similar for Dirichlet BCs. For large values of the mass transfer coefficients $k_{i}$, the moments in Figure 4 coincide with those presented in [34] for REDM.

\subsection{Reversible reaction}

This part focuses on the comparison of analytical and numerical results for reversible reactions. In the presented results, only Dirichlet BCs are considered and all parameters used in the test problems are given in Table 1.

In Figure 5, the elution profile of numerical Laplace Inversion and HR-FVS are plotted after injecting a pulse of finite width in an empty column $\left(c_{i \text {,init }}=0.0 \mathrm{gL} \mathrm{L}^{-1}\right.$ for $\left.i=1,2\right)$. In Figure $5 \mathrm{a}$, only component 1 is injected (i.e. $c_{1, \text { inj }}=1.0 \mathrm{gL}^{-1}$ and $c_{2, \text { inj }}=0.0 g \mathrm{~L}^{-1}$ ), and liquid phase reaction is assumed to be zero, while in Figure $5 \mathrm{~b}$ the injection of both components is considered (i.e. $c_{1, \text { inj }}=1.0 \mathrm{gL}^{-1}$ and $c_{2, \text { inj }}=0.5 \mathrm{gL}^{-1}$ ) and the liquid phase reaction is again neglected. On the other hand, the plots in Figures $5 \mathrm{c} \& 5 \mathrm{~d}$ show the solution profiles when reactions in both solid and liquid phases are taken into account $\left(\eta_{1}=0.05 \mathrm{~min}^{-1}, \eta_{2}=0.1 \mathrm{~min}^{-1}, \nu_{1}=0.03 \mathrm{~min}^{-1}\right.$ and $\left.\nu_{2}=0.06 \mathrm{~min}^{-1}\right)$. Good agreements in the solution profiles validate the correctness of numerical Laplace inversion and accuracy of the HR-FVS. From these results, it is again clear that the component having larger value of adsorption equilibrium constant elutes later from the column as compared to the component having smaller value of adsorption coefficient. Because of reversible reaction, the concentration levels of both components are different as compared to the irreversible reaction case shown in Figure 1. Now, larger amount of component 1 is unconverted and lesser amount of component 2 is produced. For larger values of the mass transfer coefficients $k_{i}(i=1,2)$, the solution profiles of Figure 5 become identical to those presented in [34].

Finally, a quantitative comparison of moments determined analytically, through numerical Laplace inversion and through HR-FVS is presented in Figure 6 for a wide range of flow rates considering Dirichlet BCs. Here, $c_{1, \text { inj }}=1.0 \mathrm{gL}^{-1}, c_{2, \text { inj }}=0.0 \mathrm{gL}^{-1}, c_{i, \text { init }}=0.0 \mathrm{~g} / \mathrm{l}(i=1,2), \eta_{i}=0 \mathrm{~min}^{-1}$ $(i=1,2), \nu_{1}=0.03 \mathrm{~min}^{-1}$, and $\nu_{2}=0.06 \mathrm{~min}^{-1}$. For the current reversible reaction, the magnitude of zeroth moment reflects the reduced conversion as compared to the results shown in Figure 4 describing the case of irreversible reaction. The trends in third central moments of components 1 and 2 depict that the component 1 is left skewed (left tailed) while the component 2 is right 
skewed (right tailed) which can also be seen in Figure 5. Another time, a good agreement in the results verify both the correctness of the analytical solutions and the high precision of proposed HR-FVS. The plots for Dankwerts BCs have similar behavior and are therefore omitted.

\section{Conclusion}

The two-components linear non-equilibrium model of liquid chromatography was analyzed considering first order irreversible and reversible reactions in the solid and liquid phases. The considered model was investigated for rectangular pulse injections of the reactants into an initially empty or pre-equilibrated column using two different sets of boundary conditions. The Laplace transformation and eigen-decomposition technique were jointly applied solve the model equations. The numerical Laplace inversions was used to get the desired concentration profiles in the actual time domain. Analytical temporal moments were derived from the Laplace domain solutions. These moments are helpful to investigate the amount of conversion, retention times, band broadenings, and asymmetries of the elution profiles. The analytical results were compared with the numerical results of a HR-FVS. Good agreements between the analytically and numerically determined results verified the correctness of analytical solution and the accuracy of suggested numerical algorithm. The derived analytical solutions and moments could be useful for further developments of chromatographic reactors. For instance, the analysis could be used to study the effects of mass transfer and reaction kinetics on the elution profiles, for sensitivity analysis, and for validation of the results obtained from newly introduced numerical schemes.

\section{Appendix}

\section{A Analytical moments}

Here, the analytical temporal moments are presented for two different sets of boundary conditions. For the derivation of the moments, $c_{i \text {,init }}=0 \mathrm{~g} / \mathrm{l}$ (for $\left.i=1,2\right), \eta_{i}=0$, and $c_{2, \text { inj }}=0 \mathrm{~g} / \mathrm{l}$ are considered, i.e. we are considering an empty column initially and injecting only component 1 into the reactor. Moreover, only the effect of solid phase reaction is taken into account, while the liquid phase reaction is neglected.

\section{A.1 Irreversible reaction and Dirichlet BCs}

Here, we neglect the liquid phase reaction, i.e. $\eta_{1}=0 \mathrm{~min}^{-1}$. Eqs. (60) and (61) are used to derived the moments $\mu_{n}^{(i)}$ of Laplace transformed solutions given in Eqs. (32) and (35) for $i=1,2$ and $n=0,1,2,3$. Let us define

$$
\begin{aligned}
& r=a_{1} F \widetilde{\nu}_{1} P e, \quad \gamma=\sqrt{P e\left(P e+4 a_{1} F \nu\right)}, \quad \delta_{1,2}=P e \mp \gamma, \\
& \chi_{1,2}=\frac{\gamma P e^{4}}{64}+\frac{3 \gamma^{3} P e^{2}}{32}+\frac{\gamma^{5}}{64} \mp r^{2} P e \mp \frac{3 r P e^{3}}{4} \mp \frac{P e^{5}}{8}, \\
& \chi_{3,4}:=\frac{\gamma^{3} P e}{8} \mp \frac{\gamma^{2}\left(P e^{2}+2 r\right)}{8}, \chi_{5}:=\frac{\gamma^{3}\left(P e^{2}-4 r\right)-\gamma P e^{4}}{32}, \quad \chi_{6}:=F\left(a_{1}-a_{2}\right) P e+\left(1+a_{2} F\right) r, \\
& \chi_{7,8}:=\frac{P e^{2} e^{P e}}{2}\left(\left[\frac{F\left(a_{1}-a_{2}\right) P e}{4}+\frac{r\left(1+a_{1} F\right)}{8}\right] \gamma^{3}+\frac{r P e^{2}\left(1+a_{1} F\right) \gamma}{8} \mp \frac{F\left(a_{1}-a_{2}\right) P e^{4}}{4}\right. \\
& \left.\mp \frac{3 F r\left(a_{1}-a_{2}\right) P e^{2}}{2} \mp \frac{r\left(1+a_{1} F\right)^{3} P e}{4} \mp r^{2}\left(1+a_{1} F\right) P e \mp r^{2}\left[1+\left(3 a_{1}-2 a_{2}\right) F\right]\right) .
\end{aligned}
$$

By using Eq. (60), the zeroth moments are given as

$$
\mu_{0}^{(1)}=C_{1, \text { inj }} \tau_{\text {inj }} e^{\frac{\delta_{1}}{2}}, \quad \mu_{0}^{(2)}=C_{1, \text { inj }} \tau_{\text {inj }}\left(1-e^{\frac{\delta_{1}}{2}}\right) .
$$

From Eq. (A-2), we get $\mu_{0}^{(1)}+\mu_{0}^{(2)}=C_{1, \text { inj }} \tau_{\text {inj }}$, as $C_{2, \text { inj }}=0$ is considered. The first moments are calculated by using the Eq. (61) for $n=1$ 


$$
\begin{aligned}
& \mu_{1}^{(1)}=\frac{\tau_{\text {inj }}}{2}+\frac{P e\left(1+a_{1} F\right)}{\gamma}, \\
& \mu_{1}^{(2)}=\frac{\tau_{\text {inj }}}{2}+\frac{P e\left[\gamma F\left(a_{2}-a_{1}\right)-r\left(1+a_{1} F\right)\right] e^{\frac{\delta_{1}}{2}}-\gamma\left[F\left(a_{2}-a_{1}\right) P e-r\left(1+a_{2} F\right)\right]}{r \gamma\left(1-e^{\frac{\delta_{1}}{2}}\right)} .
\end{aligned}
$$

342

$$
\begin{gathered}
\mu_{2}^{(1)}=\frac{\tau_{\mathrm{inj}}^{2}}{3}+\frac{P e\left(1+a_{1} F\right) \tau_{\mathrm{inj}}}{\gamma}+\frac{P e^{2}\left(1+a_{1} F\right)(\gamma+2)}{\gamma^{3}}+\frac{2 P e a_{1} F(1-\epsilon)}{\gamma \widetilde{k}_{1}}, \\
\mu_{2}^{(2)}=\frac{\tau_{\mathrm{inj}}^{2}}{3}+\left[\frac{P e\left(\gamma F\left(a_{2}-a_{1}\right)-r\left(1+a_{1} F\right)\right) e^{\frac{\delta_{1}}{2}}-\gamma\left(F\left(a_{2}-a_{1}\right) P e-r\left(1+a_{2} F\right)\right)}{r \gamma\left(1-e^{\frac{\delta_{1}}{2}}\right)}\right] \tau_{\mathrm{inj}} \\
+\frac{1}{1-e^{\frac{\delta_{1}}{2}}}\left(e ^ { \frac { \delta _ { 1 } } { 2 } } \left[-\frac{2 P e a_{1} F(1-\epsilon)}{\gamma \widetilde{k}_{1}}-\frac{2 P e F(1-\epsilon)\left(a_{1} \widetilde{k}_{2}-a_{2} \widetilde{k}_{1}\right)}{r \widetilde{k}_{1} \widetilde{k}_{2}}-\frac{2 P e^{2}\left(1+a_{1} F\right)^{2}}{\gamma^{3}}\right.\right. \\
\left.-\frac{P e^{2} F^{2}\left(a_{2}-a_{1}\right)^{2}}{r^{2}}\right]+\frac{2 P e F(1-\epsilon)\left(a_{1} \widetilde{k}_{2}-a_{2} \widetilde{k}_{1}\right)}{r \widetilde{k}_{1} \widetilde{k}_{2}}+\frac{P e^{2} F^{2}\left(a_{2}-a_{1}\right)^{2}}{r^{2}}+\frac{2\left(1+a_{2} F\right)^{2}}{P e} \\
+ \\
\left.+\frac{2 a_{2} F(1-\epsilon)}{\widetilde{k}_{2}}\right)-\frac{P e^{2}\left[\gamma F\left(a_{2}-a_{1}\right)-r\left(1+a_{1} F\right)\right]^{2} e^{\frac{\delta_{1}}{2}}-\gamma^{2}\left[F\left(a_{2}-a_{1}\right) P e-r\left(1+a_{2} F\right)\right]^{2}}{r^{2} \gamma^{2}\left(1-e^{\frac{\delta_{1}}{2}}\right)} .
\end{gathered}
$$

343 The above equations are helpful to calculate the second central moments by using the given relations

$$
\mu_{2}^{\prime(i)}=\mu_{2}^{(i)}-\left(\mu_{1}^{(i)}\right)^{2}, \quad i=1,2 .
$$

344 Thus, the second central moments are given as

$$
\begin{aligned}
\mu_{2}^{\prime(1)} & =\frac{\tau_{\text {inj }}^{2}}{12}+\frac{2 P e\left[\gamma^{2} a_{1} F(1-\epsilon)+P e \widetilde{k}_{1}\left(1+a_{1} F\right)^{2}\right]}{\gamma^{3} \widetilde{k}_{1}}, \\
\mu_{2}^{\prime(2)} & =\frac{\tau_{\text {inj }}^{2}}{12}+\frac{1}{1-e^{\frac{\delta_{1}}{2}}}\left(e ^ { \frac { \delta _ { 1 } } { 2 } } \left[-\frac{2 P e a_{1} F(1-\epsilon)}{\gamma \widetilde{k}_{1}}-\frac{2 P e F(1-\epsilon)\left(a_{1} \widetilde{k}_{2}-a_{2} \widetilde{k}_{1}\right)}{r \widetilde{k}_{1} \widetilde{k}_{2}}-\frac{2 P e^{2}\left(1+a_{1} F\right)^{2}}{\gamma^{3}}\right.\right. \\
& \left.-\frac{P e^{2} F^{2}\left(a_{2}-a_{1}\right)^{2}}{r^{2}}\right]+\frac{2 P e F(1-\epsilon)\left(a_{1} \widetilde{k}_{2}-a_{2} \widetilde{k}_{1}\right)}{r \widetilde{k}_{1} \widetilde{k}_{2}}+\frac{P e^{2} F^{2}\left(a_{2}-a_{1}\right)^{2}}{r^{2}}+\frac{2\left(1+a_{2} F\right)^{2}}{P e} \\
& \left.+\frac{2 a_{2} F(1-\epsilon)}{\widetilde{k}_{2}}\right)-\left[\frac{P e^{2}\left[\gamma F\left(a_{2}-a_{1}\right)-r\left(1+a_{1} F\right)\right]^{2} e^{\frac{\delta_{1}}{2}}-\gamma^{2}\left[F\left(a_{2}-a_{1}\right) P e-r\left(1+a_{2} F\right)\right]^{2}}{r^{2} \gamma^{2}\left(1-e^{\frac{\delta_{1}}{2}}\right)}\right] \\
& -\left[\frac{P e\left[\gamma F\left(a_{2}-a_{1}\right)-r\left(1+a_{1} F\right)\right] e^{\frac{\delta_{1}}{2}}-\gamma\left[F\left(a_{2}-a_{1}\right) P e-r\left(1+a_{2} F\right)\right]}{r \gamma\left(1-e^{\frac{\delta_{1}}{2}}\right)}\right]^{2} .
\end{aligned}
$$

345 The third moments are given as

$$
\begin{aligned}
& \mu_{3}^{(1)}= \frac{\tau_{\mathrm{inj}}^{3}}{4}+\frac{P e\left(1+a_{1} F\right) \tau_{\mathrm{inj}}^{2}}{\gamma}+\left[\frac{P e^{2}\left(1+a_{1} F\right)^{2}(\gamma+2)}{\gamma^{3}}+\frac{2 P e a_{1} F(1-\epsilon)}{\gamma \widetilde{k}_{1}}\right] \frac{3 \tau_{\mathrm{inj}}}{2} \\
&+\frac{P e^{3}\left(1+a_{1} F\right)^{3}\left(\gamma^{2}+6 \gamma+12\right)}{\gamma^{5}}+\frac{6 P e^{2} a_{1} F\left(1+a_{1} F\right)(\gamma+2)(1-\epsilon)}{\gamma^{3} \widetilde{k}_{1}}+ \\
&+\frac{6 P e a_{1} F(1-\epsilon)^{2}}{\gamma \widetilde{k}_{1}^{2}} . \\
& \mu_{3}^{(2)}=\frac{\tau_{\text {inj }}^{3}}{4}+\left(\frac{P e\left[\gamma F\left(a_{2}-a_{1}\right)-r\left(1+a_{1} F\right)\right] e^{\frac{\delta_{1}}{2}}-\gamma\left[F\left(a_{2}-a_{1}\right) P e-r\left(1+a_{2} F\right)\right]}{r \gamma\left(1-e^{\frac{\delta_{1}}{2}}\right)}\right) \tau_{\text {inj }}^{2}
\end{aligned}
$$




$$
\begin{aligned}
& +\frac{3 \tau_{\mathrm{inj}}}{2\left(1-e^{\frac{\delta_{1}}{2}}\right)}\left(e ^ { \frac { \delta _ { 1 } } { 2 } } \left[\frac{-2 P e^{2}\left(1+a_{1} F\right)^{2}}{\gamma^{3}}-\frac{P e^{2} F^{2}\left(a_{2}-a_{1}\right)^{2}}{r^{2}}-\frac{2 P e a_{1} F(1-\epsilon)}{\gamma \widetilde{k}_{1}}+\right.\right. \\
& \left.-\frac{2 P e F(1-\epsilon)\left(a_{1} \widetilde{k}_{2}-a_{2} \widetilde{k}_{1}\right)}{r \widetilde{k}_{1} \widetilde{k}_{2}}\right]+\frac{P e^{2} F^{2}\left(a_{2}-a_{1}\right)^{2}}{r^{2}}+\frac{2 P e F(1-\epsilon)\left(a_{1} \widetilde{k}_{2}-a_{2} \widetilde{k}_{1}\right)}{r \widetilde{k}_{1} \widetilde{k}_{2}} \\
& \left.+\frac{2\left(1+a_{2} F\right)^{2}}{P e}+\frac{2 a_{2} F(1-\epsilon)}{\widetilde{k}_{2}}\right) \\
& -\left(\frac{P e^{2}\left[\gamma F\left(a_{2}-a_{1}\right)-r\left(1+a_{1} F\right)\right]^{2} e^{\frac{\delta_{1}}{2}}-\gamma^{2}\left[F\left(a_{2}-a_{1}\right) P e-r\left(1+a_{2} F\right)\right]^{2}}{2 r^{2} \gamma^{2}\left(1-e^{\frac{\delta_{1}}{2}}\right)}\right) 3 \tau_{\text {inj }} \\
& -\frac{6 P e^{3} F^{3}\left(a_{2}-a_{1}\right)^{3}}{r^{3}}-\frac{1}{\left(1-e^{\frac{\delta_{1}}{2}}\right)}\left[\frac{P e^{3} e^{\frac{\delta_{1}}{2}}\left(1+a_{1} F\right)^{3}\left(\gamma^{2}+6 \gamma+12\right)}{\gamma^{5}}\right. \\
& -\frac{3 F P e^{3} e^{\frac{\delta_{1}}{2}}\left(1+a_{1} F\right)^{2}\left(a_{2}-a_{1}\right)(\gamma+2)}{r \gamma^{3}}+\frac{6 F^{2} P e^{3}\left(1+a_{1} F\right)\left(a_{2}-a_{1}\right)^{2}}{r^{2} \gamma} \\
& \left.+\frac{6 F\left(1+a_{2} F\right)^{2}\left(a_{2}-a_{1}\right)}{r}-\frac{\left(P e^{2}+12\right)\left(1+a_{2} F\right)^{3}}{P e^{2}}\right] \\
& +\frac{12 P e^{2} F^{2}\left(a_{2}-a_{1}\right)(1-\epsilon)\left(a_{2} \widetilde{k}_{1}-a_{1} \widetilde{k}_{2}\right)}{r^{2} \widetilde{k}_{1} \widetilde{k}_{2}}-\frac{e^{\frac{\delta_{1}}{2}} P e^{2}(1-\epsilon)}{\left(1-e^{\frac{\delta_{1}}{2}}\right)}\left[\frac{12 a_{1} F\left(1+a_{1} F\right)^{2}}{\gamma^{3} \widetilde{k}_{1}}\right. \\
& \left.-\frac{6 a_{1} F^{2}\left(a_{2}-a_{1}\right)}{r \gamma \widetilde{k}_{1}}+\frac{6 a_{1} F\left(1+a_{1} F\right)}{\gamma^{2} \widetilde{k}_{1}}-\frac{6 F\left(1+a_{1} F\right)\left(a_{2} \widetilde{k}_{1}-a_{1} \widetilde{k}_{2}\right)}{r \gamma \widetilde{k}_{1} \widetilde{k}_{2}}\right] \\
& -\frac{1}{\left(1-e^{\frac{\delta_{1}}{2}}\right)}\left[\frac{-12 a_{2} F\left(1+a_{2} F\right)(1-\epsilon)}{P e \widetilde{k}_{2}}-\frac{6 P e^{2} F^{2}\left(1+a_{2} F\right)\left(a_{2}-a_{1}\right)^{2}}{r^{2}}\right] \\
& -\frac{6 P e F(1-\epsilon)^{2}\left(a_{2} \widetilde{k}_{1}^{2}-a_{1} \widetilde{k}_{2}^{2}\right)}{r \widetilde{k}_{1}^{2} \widetilde{k}_{2}^{2}}-\frac{1}{\left(1-e^{\frac{\delta_{1}}{2}}\right)}\left[\frac{6 P e a_{1} F e^{\frac{\delta_{1}}{2}}(1-\epsilon)^{2}}{\gamma \widetilde{k}_{1}^{2}}+\frac{6 P e a_{2} F^{2}(1-\epsilon)\left(a_{2}-a_{1}\right)}{r \widetilde{k}_{2}}\right. \\
& \left.+\frac{3 P e F\left(1+a_{2} F\right)^{2}\left(a_{2}-a_{1}\right)}{r}+\frac{6 P e F(1-\epsilon)\left(1+a_{2} F\right)\left(a_{2} \widetilde{k}_{1}-a_{1} \widetilde{k}_{2}\right)}{r \widetilde{k}_{1} \widetilde{k}_{2}}\right] \\
& -\frac{1}{\left(1-e^{\frac{\delta_{1}}{2}}\right)}\left[\frac{-6\left(1+a_{2} F\right)^{3}}{P e}-\frac{6 a_{2} F(1-\epsilon)^{2}}{\widetilde{k}_{2}^{2}}-\frac{6 a_{2} F(1-\epsilon)\left(1+a_{2} F\right)}{\widetilde{k}_{2}}\right] .
\end{aligned}
$$

Finally, the third central moments can be deduced from the given relations

$$
\mu_{3}^{\prime(i)}=\mu_{3}^{(i)}-3 \mu_{1}^{(i)} \mu_{2}^{(i)}+2\left(\mu_{1}^{(i)}\right)^{3}, \quad i=1,2 .
$$

The expressions of third central moments were very lengthy. Therefore, only plots of these moments are shown in the test problems.

\section{A.2 Irreversible reaction with Danckwerts BCs}

Here, the moments are derived of the solutions given in Eqs. (40) and (41).

The zeroth moments are given as

$$
\mu_{0}^{(1)}=\frac{-4 C_{1, \mathrm{inj}} \tau_{\mathrm{inj}} \gamma P e e^{P e}}{\delta_{1}^{2} e^{\frac{\delta_{1}}{2}}-\delta_{2}^{2} e^{\frac{\delta_{2}}{2}}}, \quad \mu_{0}^{(2)}=C_{1, \mathrm{inj}} \tau_{\mathrm{inj}}\left[1+\frac{4 \gamma P e e^{P e}}{\delta_{1}^{2} e^{\frac{\delta_{1}}{2}}-\delta_{2}^{2} e^{\frac{\delta_{2}}{2}}}\right]
$$

From Eq. (A-13), it follows that $\mu_{0}^{(1)}+\mu_{0}^{(2)}=C_{1, \text { inj }} \tau_{\text {inj }}$, as $C_{2, \text { inj }}=0$ is considered.

The first moments take the form

$$
\begin{gathered}
\mu_{1}^{(1)}=\frac{\tau_{\text {inj }}}{2}-\frac{2 P e\left(1+a_{1} F\right)}{\gamma^{2}\left(\delta_{1}^{2} e^{\frac{\delta_{1}}{2}}-\delta_{2}^{2} e^{\frac{\delta_{2}}{2}}\right)}\left[\gamma\left(2 r+P e^{2}\right)\left(e^{\frac{\delta_{1}}{2}}+e^{\frac{\delta_{2}}{2}}\right)-\left(4 r(1+P e)+P e^{3}\right)\left(e^{\frac{\delta_{1}}{2}}-e^{\frac{\delta_{2}}{2}}\right)\right] . \\
\mu_{1}^{(2)}=\frac{32 \tau_{\text {inj }}\left(\chi_{1} e^{\delta_{1}}+\left[\chi_{5} e^{\frac{\delta_{2}}{2}}-P e e^{P e} \chi_{3}\right] e^{\frac{\delta_{1}}{2}}+\left[\chi_{2} e^{\frac{\delta_{2}}{2}}-P e e^{P e} \chi_{4}\right] e^{\frac{\delta_{2}}{2}}\right)}{\gamma\left(\delta_{1}^{2} e^{\frac{\delta_{1}}{2}}-\delta_{2}^{2} e^{\frac{\delta_{2}}{2}}\right)\left(\delta_{1}^{2} e^{\frac{\delta_{1}}{2}}-\delta_{2}^{2} e^{\frac{\delta_{2}}{2}}+4 \gamma P e e^{P e}\right)}
\end{gathered}
$$




$$
+\frac{64\left[\chi_{1} \chi_{6} e^{\delta_{1}}+\left[\chi_{5} \chi_{6} e^{\frac{\delta_{2}}{2}}-\chi_{7}\right] e^{\frac{\delta_{1}}{2}}+\left[\chi_{2} \chi_{6} e^{\frac{\delta_{2}}{2}}-\chi_{8}\right] e^{\frac{\delta_{2}}{2}}\right]}{r \gamma\left(\delta_{1}^{2} e^{\frac{\delta_{1}}{2}}-\delta_{2}^{2} e^{\frac{\delta_{2}}{2}}\right)\left(\delta_{1}^{2} e^{\frac{\delta_{1}}{2}}-\delta_{2}^{2} e^{\frac{\delta_{2}}{2}}+4 \gamma P e e^{P e}\right)} .
$$

The second and third central moments are not given here due to their lengthy expressions.

\section{A.3 Reversible reaction with Dirichlet BCs}

The Eqs. (60) and (61) are used to derived the moments $\mu_{n}^{(i)}$ of Laplace transformed solutions given in Eqs. (53) and (54) for $i=1,2$ and $n=0,1,2,3$. Let us define

$$
\begin{aligned}
& R_{1}=\left(a_{1} \widetilde{\nu}_{1}+a_{2} \widetilde{\nu}_{2}\right) F P e, \quad \gamma_{1,2}=\sqrt{P e^{2}+2 R_{1} \mp 2 \sqrt{P+R_{1}^{2}}}, \quad \delta_{1,2}=P e \mp \gamma_{1} \\
& \delta_{3,4}=P e \mp \sqrt{P e^{2}+2 R_{1}+2 \sqrt{P+R_{1}^{2}}}, \quad \chi_{1,2}:=W \mp \sqrt{P+R_{1}^{2}}, \\
& \chi_{3}=2\left(P-W^{2}+R_{1}^{2}\right)\left\{\left(a_{1} F+a_{2}\right) \epsilon-a_{2}\right\} \gamma_{1}+\left\{\left(2+a_{1} F-a_{2}\right) \epsilon+a_{2}\right\}\left(P+R_{1}^{2}\right)^{\frac{3}{2}} \\
& +W\left[W\left\{\left(a_{1} F+a_{2}\right) \epsilon-a_{2}\right\} \sqrt{P+R_{1}^{2}}-2 \epsilon\left(P+R_{1}^{2}\right)\left(1+a_{1} F\right)\right], \\
& \chi_{4}=-2\left(P-W^{2}+R_{1}^{2}\right)\left\{\left(a_{1} F+a_{2}\right) \epsilon-a_{2}\right\} \gamma_{2}+\left\{\left(2+a_{1} F-a_{2}\right) \epsilon+a_{2}\right\}\left(P+R_{1}^{2}\right)^{\frac{3}{2}} \\
& +W\left[W\left\{\left(a_{1} F+a_{2}\right) \epsilon-a_{2}\right\} \sqrt{P+R_{1}^{2}}+2 \epsilon\left(P+R_{1}^{2}\right)\left(1+a_{1} F\right)\right], \\
& \chi_{5}=-2 W\left\{\left(a_{1} F+a_{2}\right) \epsilon-a_{2}\right\} \gamma_{1}+W\left\{\left(a_{2}+a_{1} F\right) \epsilon-a_{2}\right\} \sqrt{P+R_{1}^{2}} \\
& -\left(P+R_{1}^{2}\right)\left\{\left(2-a_{2}+a_{1} F\right) \epsilon+a_{2}\right\}, \\
& \chi_{6}=2 W\left\{\left(a_{1} F+a_{2}\right) \epsilon-a_{2}\right\} \gamma_{2}+W\left\{\left(a_{2}+a_{1} F\right) \epsilon-a_{2}\right\} \sqrt{P+R_{1}^{2}} \\
& +\left(P+R_{1}^{2}\right)\left\{\left(2-a_{2}+a_{1} F\right) \epsilon+a_{2}\right\} .
\end{aligned}
$$

Using Eq. (60), the zeroth moments can be calculated as

$$
\mu_{0}^{(1)}=\frac{C_{1, \text { inj }} \tau_{\text {inj }}\left(\chi_{2} e^{\frac{\delta_{3}}{2}}-\chi_{1} e^{\frac{\delta_{1}}{2}}\right)}{2 \sqrt{P+R_{1}^{2}}}, \quad \mu_{0}^{(2)}=\frac{r_{3} C_{1, \text { inj }} \tau_{\text {inj }}}{\sqrt{P+R_{1}^{2}}}\left(e^{\frac{\delta_{1}}{2}}-e^{\frac{\delta_{3}}{2}}\right)
$$

The first moments are derived by using Eq. (61) for $n=1$

$$
\begin{aligned}
& \mu_{1}^{(1)}=\frac{\tau_{\text {inj }}}{2}+\frac{P e\left(\gamma_{2} \chi_{3} e^{\frac{\delta_{1}}{2}}+\gamma_{1} \chi_{4} e^{\frac{\delta_{3}}{2}}\right)}{2 \epsilon \gamma_{1} \gamma_{2}\left(P+R_{1}^{2}\right)\left(\chi_{2} e^{\frac{\delta_{3}}{2}}-\chi_{1} e^{\frac{\delta_{1}}{2}}\right)}, \\
& \mu_{1}^{(2)}=\frac{\tau_{\text {inj }}}{2}+\frac{P e\left(\gamma_{2} \chi_{5} e^{\frac{\delta_{1}}{2}}+\gamma_{1} \chi_{6} e^{\frac{\delta_{3}}{2}}\right)}{2 \epsilon \gamma_{1} \gamma_{2}\left(P+R_{1}^{2}\right)\left(e^{\frac{\delta_{3}}{2}}-e^{\frac{\delta_{1}}{2}}\right)} .
\end{aligned}
$$

Moreover, the expressions of analytical $\mu_{2}^{\prime(i)}$ and $\mu_{3}^{\prime(i)}$ were very lengthy. Therefore, plots of second and third central moments are shown in Figure 6.

A.4 Reversible reaction with Danckwerts BCs

Here, the moments are derived of the solutions given in Eqs. (58) and (59). The zeroth moments are

$$
\begin{aligned}
& \mu_{0}^{(1)}=\frac{2 C_{1, \mathrm{inj}} \tau_{\mathrm{inj}} P e e^{P e}}{\sqrt{P+R_{1}^{2}}}\left[\frac{\chi_{1} \gamma_{1}}{\delta_{1}^{2} e^{\frac{\delta_{1}}{2}}-\delta_{2}^{2} e^{\frac{\delta_{2}}{2}}}-\frac{\chi_{2} \gamma_{2}}{\delta_{3}^{2} e^{\frac{\delta_{3}}{2}}-\delta_{4}^{2} e^{\frac{\delta_{4}}{2}}}\right], \\
& \mu_{0}^{(2)}=\frac{-4 r_{3} C_{1, \mathrm{inj}} \tau_{\mathrm{inj}} P e e^{P e}}{\sqrt{P+R_{1}^{2}}}\left[\frac{\gamma_{1}}{\delta_{1}^{2} e^{\frac{\delta_{1}}{2}}-\delta_{2}^{2} e^{\frac{\delta_{2}}{2}}}-\frac{\gamma_{2}}{\delta_{3}^{2} e^{\frac{\delta_{3}}{2}}-\delta_{4}^{2} e^{\frac{\delta_{4}}{2}}}\right] .
\end{aligned}
$$


Other moments are not presented here due to their lengthy expressions.

\section{Compliance with ethical standards}

Conflict of interest: The authors declare that they have no conflict of interest.

Ethical approval: This article does not contain any studies with human participants or animals performed by any of the authors.

\section{References}

1. Ganetsos, G., Barker, P.E.: Preparative and production scale chromatography. Vol. 61, Marcel Dekker, Inc., New York, 375-523, (1993).

2. Guiochon, G., Lin, B.: Modeling for preparative chromatography. Academic Press, (2003).

3. Guiochon, G., Shirazi, G., Katti, M.: in: Fundamentals of preparative and nonlinear chromatography, 2nd ed., Elsevier Inc., Netherlands, (2006).

4. Mazzotti, M., Kruglov, A., Neri, B., Gelosa, D., Morbidelli, M.: A continuous chromatographic reactor: SMBR Chem. Eng. Sci. 51, 1827-1836, (1996).

5. Yamaoka, K., Nakagawa, T.: Moment analysis for reaction chromatography. J. Chromatogr. A 117, 1-10, (1976).

6. Takeuchi, K., Uraguchi, Y.: Separation conditions of the reactant and the product with a chromatographic moving bed reactor. J. Chem. Eng. Japan 9, 164-166, (1976).

7. Takeuchi, K., Uraguchi, Y.: Basic design of chromatographic moving-bed reactors for product refining. J. Chem. Engng Japan 9, 246-248, (1976).

8. Takeuchi, K., Uraguchi, Y.: The effect of the exhausting section on the performance of a chromatographic moving-bed reactor. J. Chem. Engng Japan 10, 72-74, (1977).

9. Takeuchi, K., Uraguchi, Y.: Experimental studies of a chromatographic moving-bed reactor-catalytic oxidation of carbon monoxide on activated alumina as a model reactor. J. Chem. Eng. Japan 10, 455-460, (1977).

10. Takeuchi, K., Miyauchi, T., Uraguchi, Y.: Computational studies of a chromatographic moving bed reactor for consecutive and reversible reactions. J. Chem. Eng. Japan 11, 216-220, (1978).

11. Schweich, D., Villermaux, J.: The chromatographic reactor. A new theoretical approach. Ind. Eng. Chem. Fundamen. 17, 1-7, (1978).

12. Villermaux, J.: The chromatographic reactor, in percolation processes: Theory and applications. A.E. Rodrigues and D. Tondeur (Eds.), Sijthoff \& Noordhoff, Maryland, (1981).

13. Cho, B.K., Aris, R., Carr, R.W.: The mathematical theory of a countercurrent catalytic reactor. Proc. R. Sot. Lond. 383, 147-189, (1982).

14. Petroulas, T., Aris, R., Carr, R.W.: Analysis of the counter-current moving-bed chromatographic reactor, Comp. Math. Appl. 11, 5-34, (1985).

15. Carta, G.: Simultaneous reaction and chromatography, in: Chromatographic and membrane processes in biotechnology (Edited by C. A. Costa and J. S. Cabral), 429-447, NATO ASI, Kluwer Academic Publishers, Deventer, (1991).

16. Binous, H., McCoy, B.: Chromatographic reactions of the three components: Application to separations. Chem. Eng. Sci. 47, 4333-4343, (1992).

17. Sardin, M., Schweich, D., Villermaux, J., Ganetsos, G., Barker, P.E. (Eds.): Preparative fixed-bed chromatographic reactor, Preparative and production scale chromatography, Marcel Dekker Inc., New York, USA, (1993).

18. Lin, B., Song, F., Guiochon, G.: Analytical solution of the ideal, nonlinear model of reaction chromatography for a reaction $\mathrm{A} \leftarrow \mathrm{B}$ and a parabolic isotherm. J. Chromatogr. A 1003, 91-100, (2003).

19. Borren, T., Fricke, J., Schmidt-Traub, H. (Eds.): Chromatographic reactors in preparative chromatography of fine chemicals and pharmaceutical agents. Wiley-VCH Verlag, Weinheim, 371-395, (2005).

20. Fricke, J., Schmidt-Traub, H., Kawase, M.: Chromatographic reactor. Ullmann's Encyclopedia of Industrial Chemistry, Wiley-VCH Verlag, Weinheim, (2005).

21. Javeed, S., Qamar, S., Seidel-Morgenstern, A., Warnecke, G.: A discontinuous Galerkin method to solve chromatographic models, J. Chromatogr. A 1218, 7137-7146, (2011).

22. Javeed, S., Qamar, S., Seidel-Morgenstern, A., Warnecke, G.: Parametric study of thermal effects in reactive liquid chromatography. Chem. Eng. J. 191, 426-440, (2012).

23. Qamar, S., Bibi, S., Khan, F.U., Shah, M., Javeed, S., Seidel-Morgenstern, A.: Irreversible and reversible reactions in a liquid chromatographic column: analytical solutions and moment analysis, Ind. Eng. Chem. res. 53, 2461-2472, (2014).

24. Ruthven, D.M.: Principles of adsorption and adsorption processes. Wiley-Interscience, New York, (1984).

25. Van Genuchten, M.Th.: Analytical solutions for chemical transport with simultaneous adsorption, zeroth-order production and first order decay. J. Hydrology 49, 213-233, (1981).

26. Rice, R.G., Do, D.D.: Applied mathematics and modeling for chemical engineers, Wiley-Interscience, New York, (1995).

27. Durbin, F.: Numerical Inversion of Laplace Transforms: An efficient improvement to Dubner and Abate's Method. The Computer Journal 17, 371-376, (1974). 
28. Schneider, P., Smith, J.M.: Adsorption rate constants from chromatography, AIChE J. 14, 762-771, (1968).

29. Mehta, R.V., Merson, R.L., McCoy, B.J.: Hermite polynomial representation of chromatography elution curves. J. Chromatography A 88, 1-6, (1974).

30. Linek, F., Duduković, M.P.: Representation of breakthrough curves for fixed-bed adsorbers and reactors using moments of the impulse response. Chem. Eng. J. 23, 31-36, (1982).

31. Javeed, S., Qamar, S., Ashraf, W., Warnecke, G., Seidel-Morgenstern, A.: Analysis and numerical investigation of two dynamic models for liquid chromatography, Chem. Eng. Sci. 90, 17-31, (2013).

32. Qamar, S., Abbasi, J.N., Javeed, S., Shah, M., Khan, F.U., Seidel-Morgenstern, A.: Analytical solutions and moment analysis of chromatographic models for rectangular pulse injections. J. Chromatogr. A 1315, 92-106, (2013).

33. Suzuki, M., Smith, J.M.: Kinetic studies by chromatography. Chem. Eng. Sci. 26 221-235, (1971).

34. Bibi, S., Qamar, S., Seidel-Morgenstern, A.: Irreversible and reversible reactive chromatography: analytical solutions and moment analysis for rectangular pulse injections. J. Chromatogr. A 1385, 49-62, (2015).

35. Quezada, C.R., Clement, T.P., Lee, K.K.: Generalized solution to multi-dimensional multi-species transport equations coupled with a first-order reaction network involving distinct retardation factors. Advances in water resources $27,507-520,(2004)$

36. Abramowitz, M., Stegun, I.A.: Handbook of mathematical functions. Dover, New York, N.Y, (1970).

37. Carslaw, H.S., Jaeger, J.D.: Conduction of heat in solids. Oxford Unversity Press, London, 2nd ed, (1959).

38. Javeed, S., Qamar, S., Seidel-Morgenstern, A., Warnecke, G.: Efficient and accurate numerical simulation of nonlinear chromatographic processes. J. Comput. Chem. Eng. 35, 2294-2305, (2011).

39. Danckwerts, P.V.: Continuous flow systems. Chem. Eng. Sci. 2, 1-9, (1953).

40. Koren, B.: A robust upwind discretization method for advection, diffusion and source terms. In C. B. Vreugdenhil, B. Koren, editors, Numerical Methods for Advection-Diffusion Problems, Volume 45 of Notes on Numerical Fluid Mechanics, chapter 5, pages 117-138, Vieweg Verlag, Braunschweig, (1993).

41. Trapp, O., Schurig, V.: ChromWin A computer program for the determination of enantiomerization barriers in dynamic chromatography. Comput. Chem. 25, 187-195, (2001).

42. Trapp, O., Trapp, G., Schurig, V.: Direct calculation and computer simulation of the enantiomerization barrier of oxazepam in dynamic HPLC experiments-a comparative study. J. Biochem. Biophys. Methods 54, 301-313, (2002).

Table 1 Parameters for two-component RLKM.

\begin{tabular}{|c|c|}
\hline Parameters & values \\
\hline Length of column & $L_{\max }=25.0 \mathrm{~cm}$ \\
Diameter of column & $d=0.46 \mathrm{~cm}$ \\
Porosity & $\epsilon=0.35$ \\
Mobile phase velocity & $u=3.0 \mathrm{~cm}\left(\mathrm{~min}^{-1}\right)$ \\
Time of injection & $t_{\mathrm{inj}}=10 \mathrm{~min}^{-}$ \\
Peclet number & $P e=500$ \\
$c_{i, \text { init }}=0 \mathrm{gL}^{-1}$ \\
Initial concentration of $i$-th component & $a_{1}=2.0$ \\
Adsorption equilibrium constant for component 1 & $a_{2}=0.5$ \\
Adsorption equilibrium constant for component 2 & $k_{1}=1 \mathrm{~min}^{-1}$ \\
Mass transfer coefficient for component 1 & $k_{2}=1 \mathrm{~min}^{-1}$ \\
Mass transfer coefficient for component 2 & $\eta_{1}=0.05 \mathrm{~min}^{-1}$ \\
Liquid phase reaction rate constant (component 1) & $\eta_{2}=0.1 \mathrm{~min}^{-1}$ \\
Liquid phase reaction rate constant (component 2) & $\nu_{1}=0.03 \mathrm{~min}^{-1}$ \\
Solid phase reaction rate constant (component 1) & $\nu_{2}=0.06 \mathrm{~min}^{-1}$ \\
Solid phase reaction rate constant (component 2) &
\end{tabular}



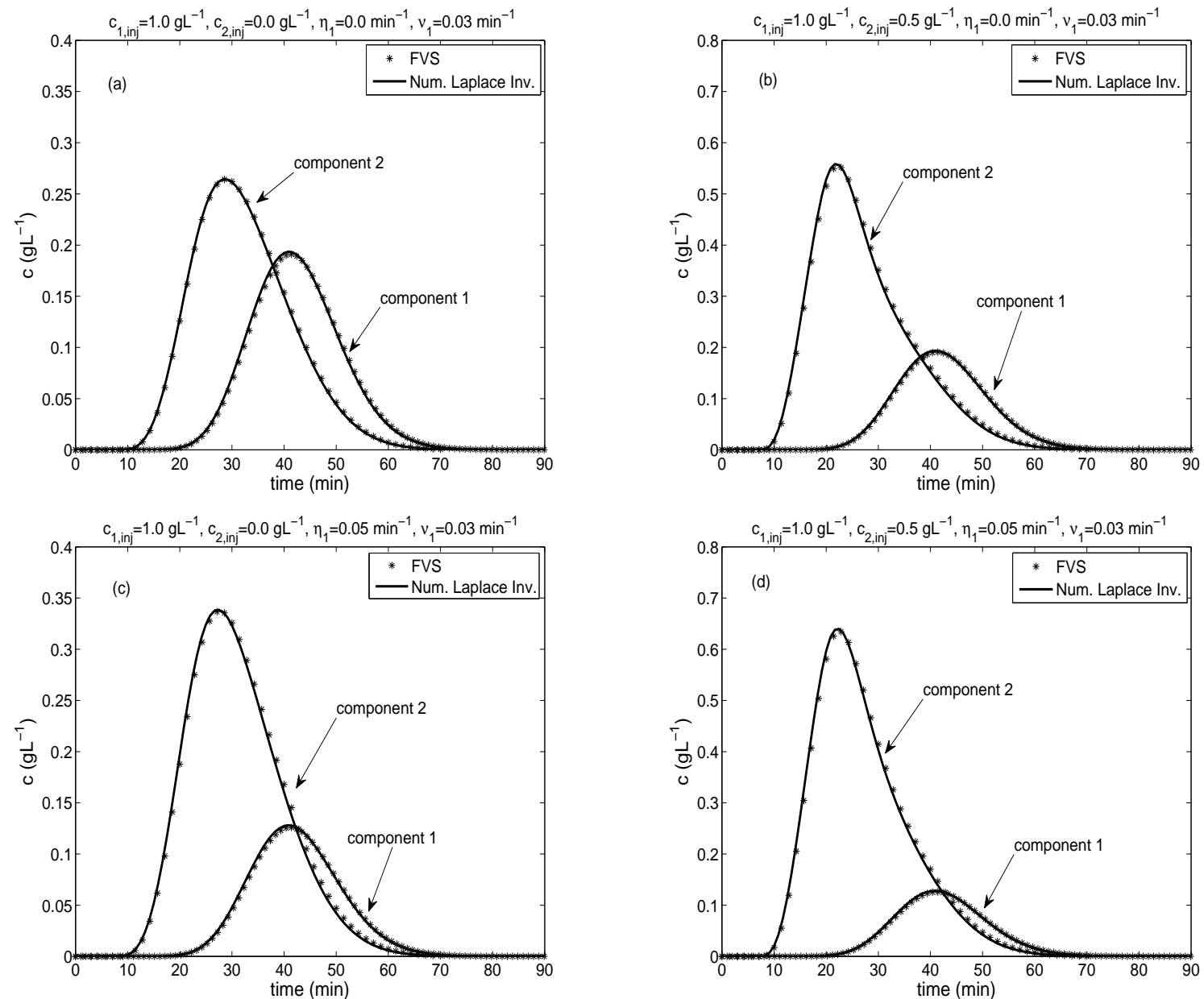

Fig. 1 Irreversible reaction: Plots (a)\&(b): Solutions for Dirichlet BCs at $x=1$ with zero liquid phase reaction. Plots (c)\&(d): Comparison of solutions at $x=1$ for both solid and liquid phases reactions using Dirichlet BCs. Here, $c_{i \text {,init }}=0 g L^{-1}(i=1,2)$ and other parameters are given in Table 1.
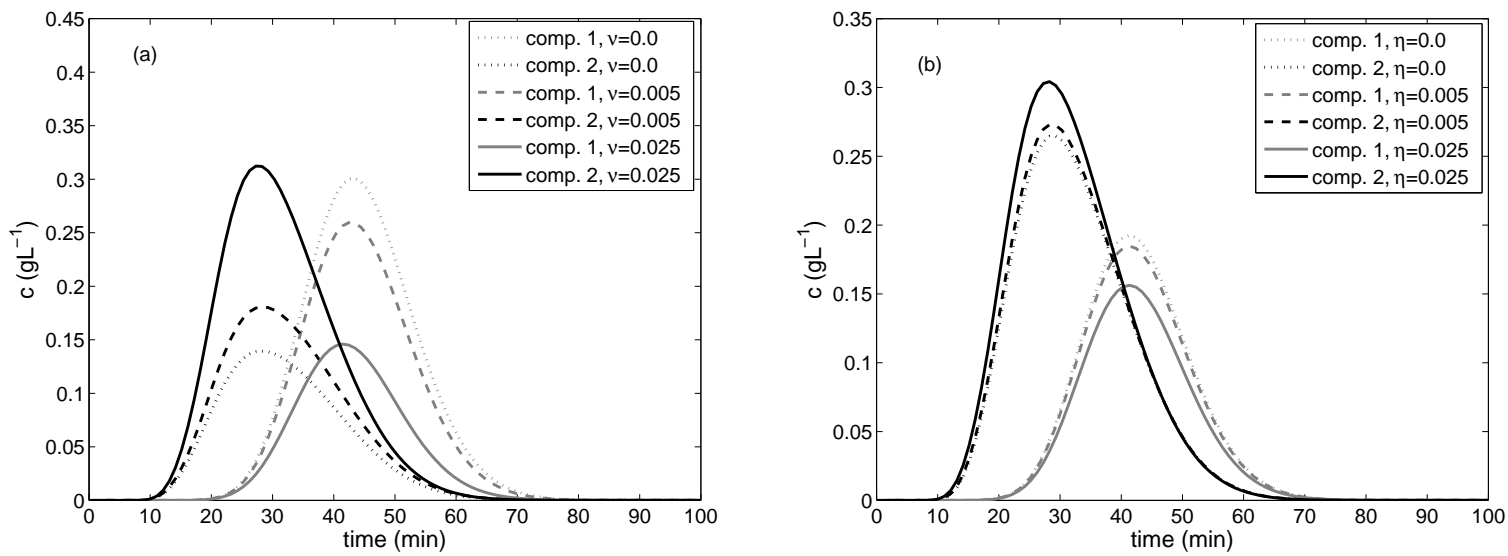

Fig. 2 Irreversible reaction: Plot (a): Concentration profiles at $x=1$ for different values of the solid phase reaction rate constant. Plot (b): Concentration profiles at $x=1$ for different values of liquid phase reaction rate constant. Other parameters are given in Table 1. 

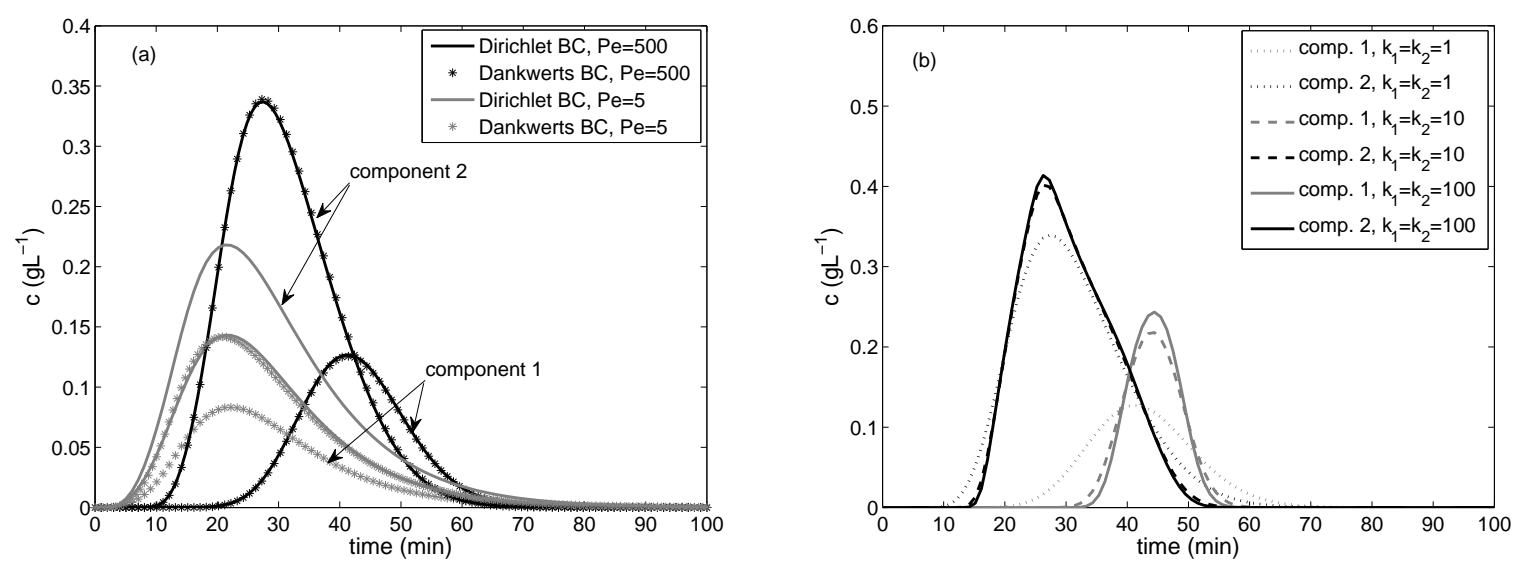

Fig. 3 Irreversible reaction: Plot (a): Effect of BCs for different Peclet numbers with $c_{1, \text { inj }}=1 g L^{-1}, c_{2, \text { inj }}=0 g L^{-1}$ and $c_{i \text {,init }}=0 \mathrm{gL}^{-1}$ for $i=1,2$. Plot (b): Effect of mass transfer coefficient on the concentration profiles. Other parameters are given in Table 1.
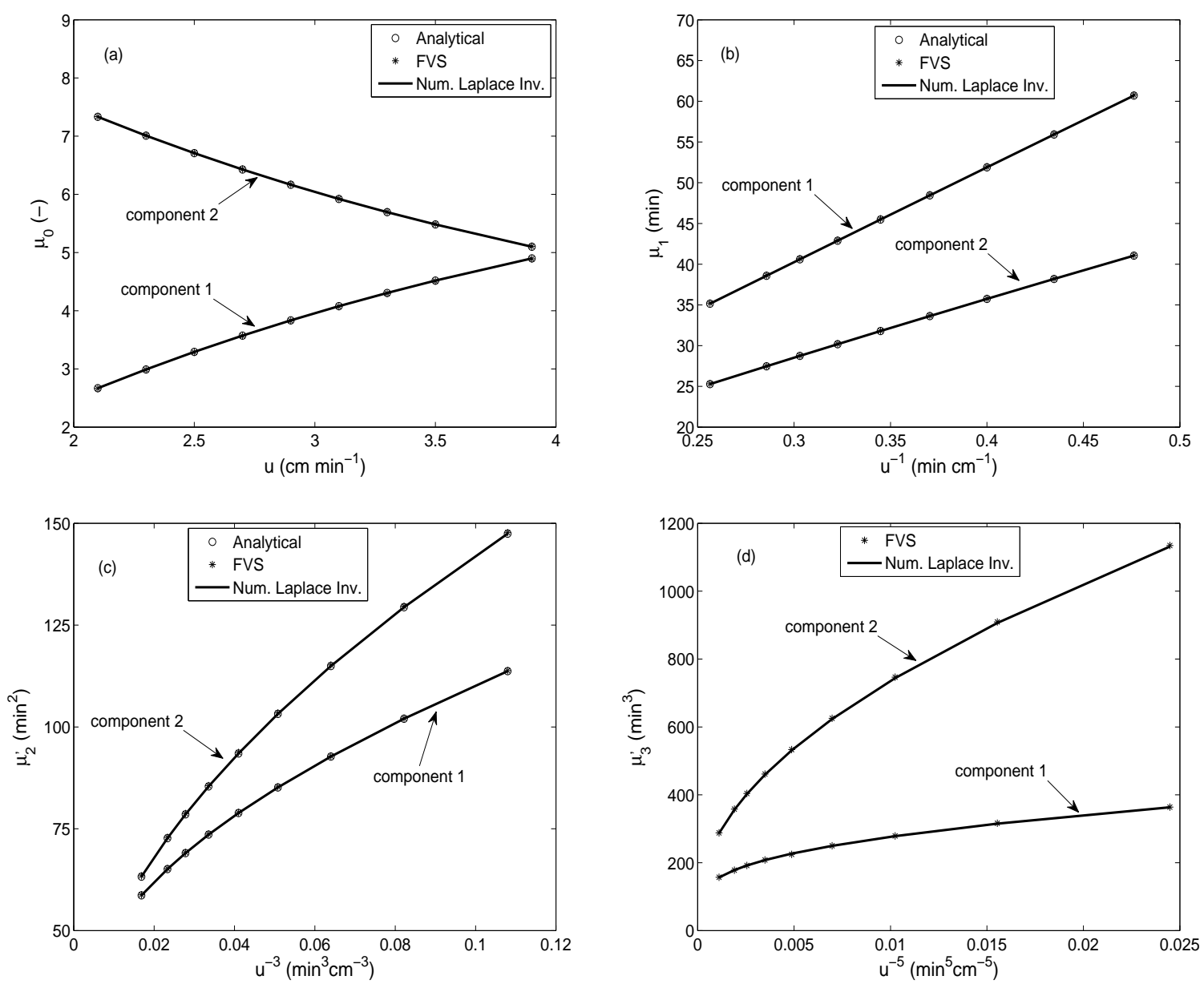

Fig. 4 Moments of irreversible reaction with Dirichlet BCs considering different values of $u$ for $\eta=0.0 \mathrm{~min}^{-1}$ and $\nu=0.03 \mathrm{~min}^{-1}$. Here, $c_{i \text {,init }}=0 g L^{-1}(i=1,2), c_{1, \text { inj }}=1 g L^{-1}$, and $c_{2, \text { inj }}=0 g L^{-1}$. Other parameters are listed in Table 1. 

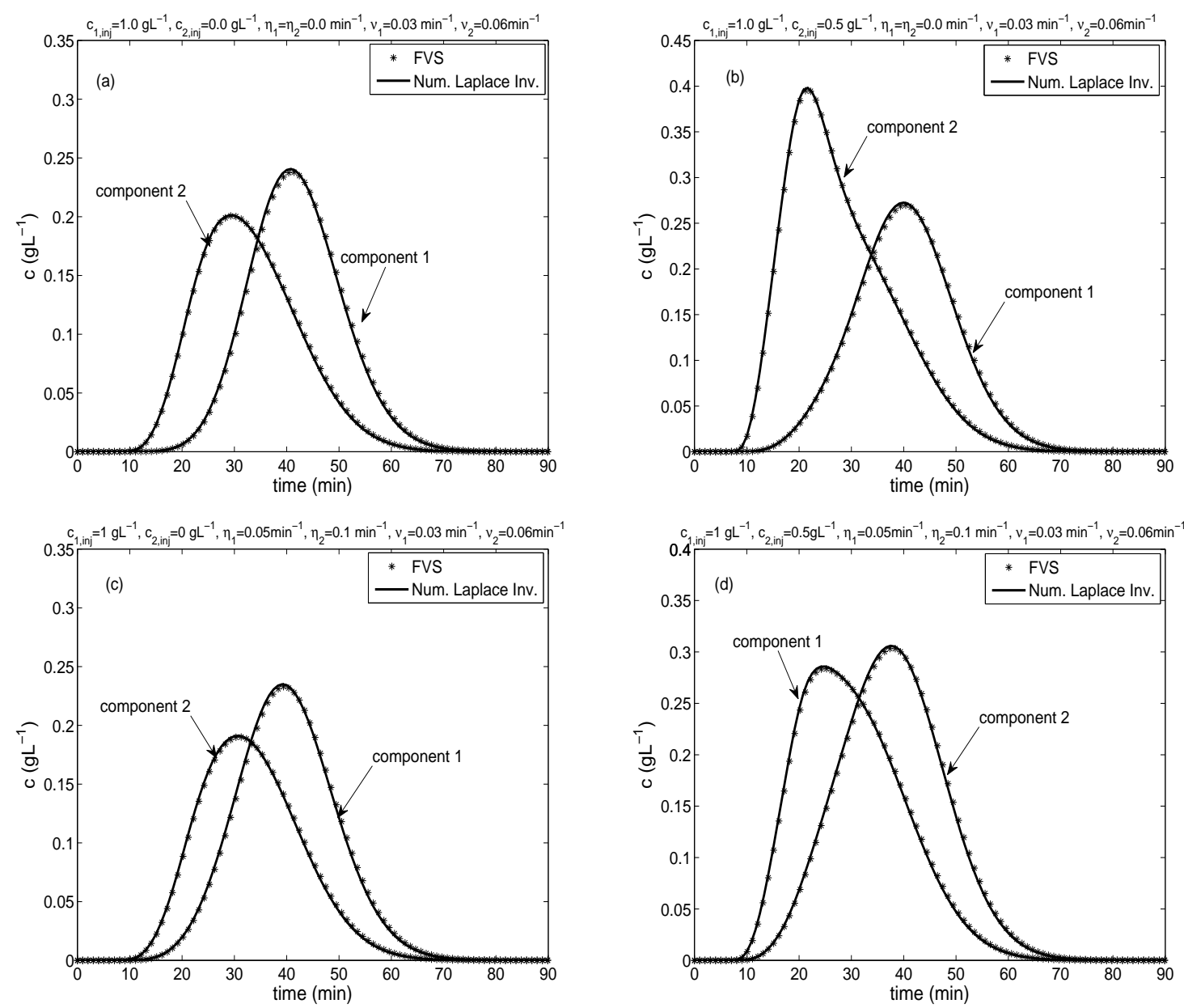

Fig. 5 Reversible reaction, Plots (a)\&(b): Solutions for Dirichlet BCs at $x=1$ with only solid phase reaction. Plots (c)\&(d): Comparison of solutions at $x=1$ for Dirichlet BCs and both liquid and solid phase reactions. Here, $c_{i, \text { init }}=0 g L^{-1}(i=1,2)$ and other parameters are given in Table 1. 

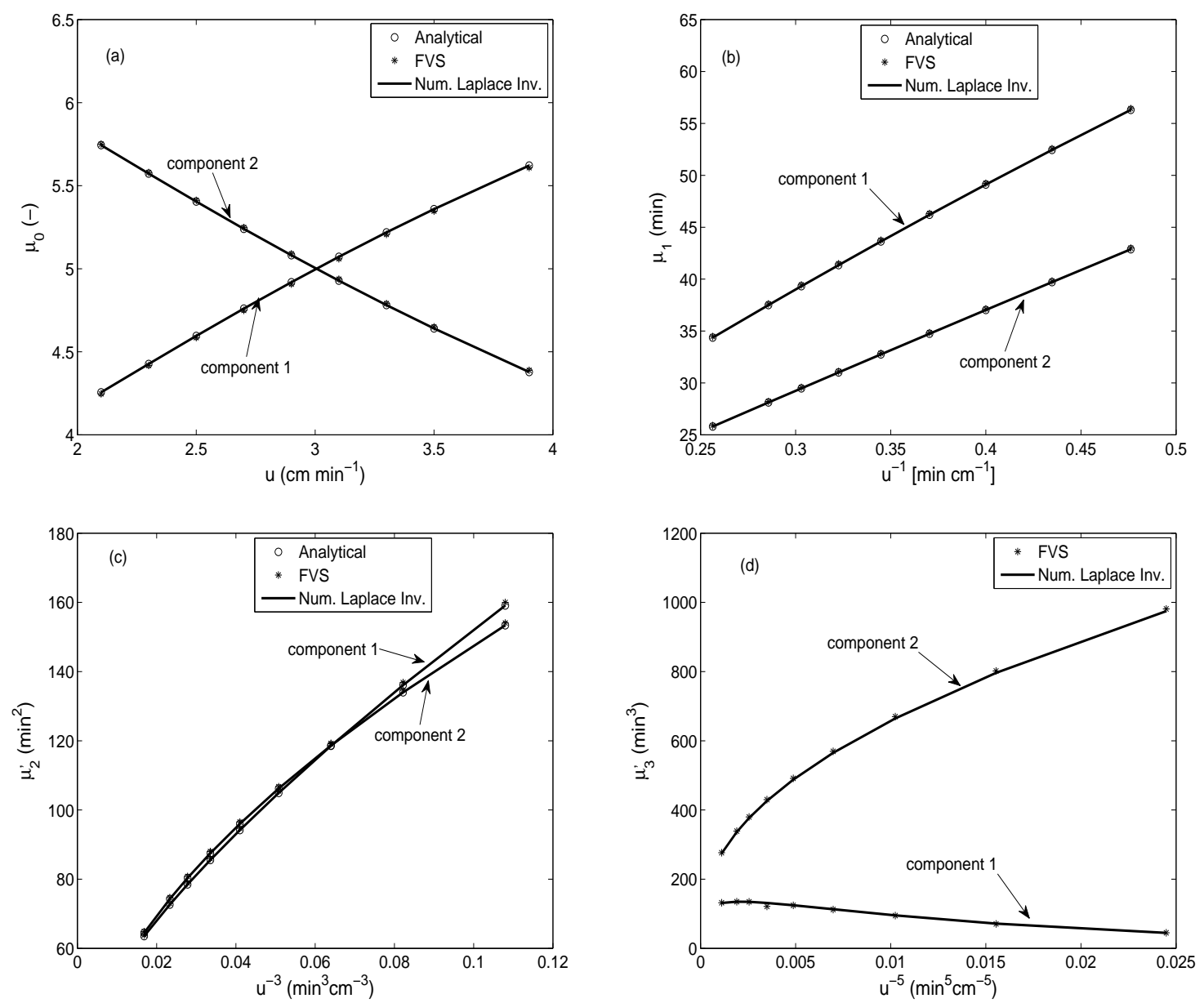

Fig. 6 Moments of reversible reaction with Dirichlet BCs considering different values of $u$ for $\eta_{1}=\eta_{2}=0 \mathrm{~min}^{-1}$, $\nu_{1}=0.03 \mathrm{~min}^{-1}, \nu_{2}=0.06 \mathrm{~min}^{-1}$. Here, $c_{i \text {,init }}=0 \mathrm{gL} \mathrm{L}^{-1}$ for $i=1,2, c_{1, \text { inj }}=1 \mathrm{gL} \mathrm{L}^{-1}$, and $c_{2, \text { inj }}=0 \mathrm{gL} L^{-1}$. Other parameters are listed in Table 1. 